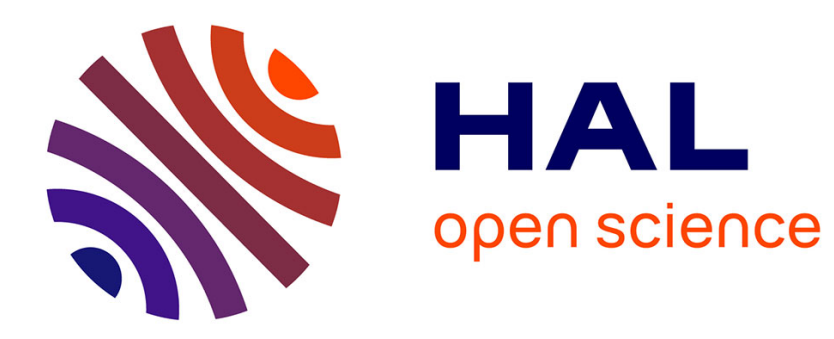

\title{
Surface waves in elastic half spaces coated with crystalline films
}

David Steigmann

\section{To cite this version:}

David Steigmann. Surface waves in elastic half spaces coated with crystalline films. 2013. hal00783078

\section{HAL Id: hal-00783078 \\ https://hal.science/hal-00783078}

Preprint submitted on 1 Feb 2013

HAL is a multi-disciplinary open access archive for the deposit and dissemination of scientific research documents, whether they are published or not. The documents may come from teaching and research institutions in France or abroad, or from public or private research centers.
L'archive ouverte pluridisciplinaire HAL, est destinée au dépôt et à la diffusion de documents scientifiques de niveau recherche, publiés ou non, émanant des établissements d'enseignement et de recherche français ou étrangers, des laboratoires publics ou privés. 


\title{
Surface waves in elastic half spaces coated with crystalline films
}

\author{
David J. Steigmann \\ Department of Mechanical Engineering \\ University of California \\ Berkeley, CA. 94720
}

USA

\section{Introduction}

Considered here is the general theory of surface wave propagation in elastic thin-film/substrate systems. Elasticity is of course an inherently nonlinear subject, although a great many applications are amenable to analysis using the linear theory, including those developed here. Thus for the sake of completeness and to establish the logical progression of our work we present a brief tutorial on the general nonlinear purely mechanical theory as a prelude to linearization.

The main contribution of the present work is the derivation of and solution to an asymptotic twodimensional theory for the dynamics of a thin film bonded to a substrate, as distinct from the asymptotic treatment of the underlying three-dimensional equations (Fu, 2007). Here the small parameter is the film thickness, and the considered model furnishes the rigorous leading-order system when this is small against the wavelength of a propagating surface wave.

The purely elastic theory is developed first, followed by an extension to electroelasticity. We draw particular attention to some non-standard effects associated with the propagation of Love waves in conventional isotropic elastic half spaces coated with thin films having various kinds of crystalline symmetry.

Standard notation is used throughout. Thus we use bold face for vectors and tensors and indices to denote their components. Latin indices take values in $\{1,2,3\}$; Greek in $\{1,2\}$. The latter are associated with surface coordinates and associated vector and tensor components. A dot between bold symbols is used to denote the standard inner product. Thus, if $\mathbf{A}_{1}$ and $\mathbf{A}_{2}$ are second-order tensors, then $\mathbf{A}_{1} \cdot \mathbf{A}_{2}=\operatorname{tr}\left(\mathbf{A}_{1} \mathbf{A}_{2}^{t}\right)$, where $\operatorname{tr}(\cdot)$ is the trace and the superscript ${ }^{t}$ is used to denote the transpose. The norm of a tensor $\mathbf{A}$ is $|\mathbf{A}|=\sqrt{\mathbf{A} \cdot \mathbf{A}}$. The linear operator $\operatorname{Sym}(\cdot)$ delivers the symmetric part of its second-order tensor argument. The notation $\otimes$ identifies the standard tensor product of vectors. If $\mathcal{C}$ is a fourth-order tensor, then $\mathcal{C}[\mathbf{A}]$ is the second-order tensor with orthogonal components $\mathcal{C}_{i j k l} A_{k l}$. The transpose $\mathcal{C}^{t}$ is defined by $\mathbf{B} \cdot \mathcal{C}^{t}[\mathbf{A}]=\mathbf{A} \cdot \mathcal{C}[\mathbf{B}]$, and $\mathcal{C}$ is said to possess major symmetry if $\mathcal{C}^{t}=\mathcal{C}$. If $\mathbf{A} \cdot \mathcal{C}[\mathbf{B}]=\mathbf{A}^{t} \cdot \mathcal{C}[\mathbf{B}]$ and $\mathbf{A} \cdot \mathcal{C}[\mathbf{B}]=\mathbf{A} \cdot \mathcal{C}\left[\mathbf{B}^{t}\right]$ then $\mathcal{C}$ is said to possess minor symmetry. We use symbols such as Div and Grad to denote the three-dimensional divergence and gradient operators, while div and $\nabla$ are reserved, after Section 2, for their two-dimensional counterparts. Thus, for example, $\operatorname{Div} \mathbf{A}=A_{i j, j} \mathbf{e}_{i}$ and $\operatorname{div} \mathbf{A}=A_{i \alpha, \alpha} \mathbf{e}_{i}$, where $\left\{\mathbf{e}_{i}\right\}$ is an orthonormal basis and subscripts preceded by commas are used to denote partial derivatives with respect to Cartesian coordinates. Finally, the notation $F_{\mathbf{A}}$ stands for the tensor-valued derivative of a scalar-valued function $F(\mathbf{A})$. 


\section{Brief resumé of nonlinear elasticity theory}

Background material on nonlinear elasticity theory is given in (Ciarlet, 1988; Ogden, 1997; Antman, 2005). The basic problem in this theory is to find a deformation function mapping the position $\mathbf{x}$ of a material point of a body, in some reference configuration $\kappa$, to the position $\mathbf{y}$ of the same material point of the body in its current configuration at time $t$. Thus we seek a function $\boldsymbol{\chi}(\cdot, t)$ such that $\mathbf{y}=$ $\chi(\mathbf{x}, t)$. This is presumed to be invertible at each fixed $t$, to reflect the notion that any given position may be occupied by one, and only one, material point at any instant. The inverse function theorem then requires that the deformation gradient,

$$
\mathbf{F}=\operatorname{Grad} \boldsymbol{\chi},
$$

the gradient of $\chi(\cdot, t)$ with respect to $\mathbf{x}$, be invertible.

The motion $\chi$ must be such as to satisfy the linear momentum balance

$$
\operatorname{div} \mathbf{T}+\rho \mathbf{b}=\rho \mathbf{y}_{t t},
$$

where $\mathbf{y}_{t}=\partial \boldsymbol{\chi}(\mathbf{x}, t) / \partial t$, etc., $\rho$ is the mass density in the current configuration, $\mathbf{b}$ is the body force per unit mass, $\mathbf{T}$ is the Cauchy stress, and div is the divergence operator with respect to position $\mathbf{y}$. Granted (2), the moment-of-momentum balance is simply the requirement that the Cauchy stress be symmetric, i.e.

$$
\mathbf{T}=\mathbf{T}^{t}
$$

Standard boundary data consist in the specification of $\mathbf{y}$ and the traction

$$
\mathbf{t}=\mathbf{T n}
$$

on complementary parts of the boundary, where $\mathbf{n}$ is the exterior unit normal to the bounding surface of the body in its current configuration.

For purposes of analysis it is convenient to recast (2) as a differential equation defined on the specified reference configuration $\kappa$. The relevant equation is

$$
\operatorname{Div} \mathbf{P}+\rho_{\kappa} \mathbf{b}=\rho_{\kappa} \mathbf{y}_{t t},
$$

where $\rho_{\kappa}$ is the mass density in $\kappa$, and

$$
\mathbf{P}=\mathbf{T F}^{*}
$$

is the Piola stress, in which

$$
\mathbf{F}^{*}=J \mathbf{F}^{-t}
$$

is the cofactor of the deformation gradient, with

$$
J=\operatorname{det} \mathbf{F} \text {. }
$$

Normally we denote the inverse by appending a superscript -1 ; here $-t$ is the transposed inverse, or inverted transpose, the two being equivalent by virtue of the commutativity of the inverse and transpose 
operations. If $\kappa$ is a configuration that could in principle be occupied by the material (e.g., an initial configuration), then the requirement

$$
J>0
$$

is imposed to reflect the physical requirement that matter deforms without self penetration.

The referential and current mass densities are connected by

$$
\rho_{\kappa}=\rho J
$$

and conservation of mass requires that $\rho_{\kappa}$ be independent of $t$ when expressed as a function of $\mathbf{x}$ and $t$. Accordingly it is regarded as an assigned function of $\mathbf{x}$.

The connection between the Cauchy and Piola stresses is most readily understood by expressing the force acting on an arbitrary material surface $S \subset \kappa$ in the alternative forms

$$
\int_{s} \mathbf{t} d a=\int_{S} \mathbf{p} d A
$$

where $s=\chi(S, t)$ is the image of the material surface in the current configuration, consisting of the same set of material points. Using Nanson's formula

$$
\alpha \mathbf{n}=\mathbf{F}^{*} \mathbf{N},
$$

in which $\mathbf{N}$ is the exterior unit normal to $S$ and $\alpha=\left|\mathbf{F}^{*} \mathbf{N}\right|$ is the areal stretch of $S$, we then use (4) to obtain

$$
\int_{S} \mathbf{p} d A=\int_{s} \mathbf{T} \mathbf{n} d a=\int_{S} \mathbf{T F}^{*} \mathbf{N} d A
$$

and hence

$$
\mathbf{p}=\mathbf{P N}
$$

Thus the Piola stress operates on the referential unit normal to furnish the force per unit reference area.

To model elastic bodies we assume the stress $\mathbf{T}$ ( or $\mathbf{P}$ ) to be given by an empirical function of $\mathbf{F}$, which may depend parametrically on $\mathbf{x}$ if the material properties are non-uniform, as in a functionally graded material. Thermodynamic considerations pertaining to the non-existence of perpetual motion machines imply that the stress is determined via an empirical strain-energy function $W(\mathbf{F})$, i.e. (Ogden, 1997)

$$
\mathbf{P}=W_{\mathbf{F}}
$$

the gradient of $W$ with respect to $\mathbf{F}$. This too depends parametrically on $\mathbf{x}$ in non-uniform materials. Here, however, we are concerned exclusively with uniform materials, for which there is no such dependence.

Combining (6) with (15) we conclude that the function $W$ must be such that $\left(W_{\mathbf{F}}\right) \mathbf{F}^{t}$ is symmetric; that is, equal to its own transpose. This in turn is equivalent to

$$
\left(W_{\mathbf{F}}\right) \mathbf{F}^{t} \cdot \boldsymbol{\Omega}=0
$$

for all skew tensors $\boldsymbol{\Omega}\left(\boldsymbol{\Omega}^{t}=-\boldsymbol{\Omega}\right)$. The symmetries inherent in the dot product imply that (16) is equivalent to

$$
W_{\mathbf{F}} \cdot \Omega \mathbf{F}=0
$$


Fix $\boldsymbol{\Omega}$ and consider the one-parameter family of tensors $\mathbf{Q}(u)$ defined by the initial-value problem

$$
\dot{\mathbf{Q}}=\mathbf{\Omega} \mathbf{Q} \quad \text { with } \quad \mathbf{Q}(0)=\mathbf{I},
$$

where $\mathbf{I}$ is the identity tensor and the superposed dot is the derivative with respect to $u$. The components of the identity are simply the Kronecker deltas $\delta_{i j}$. It is well known that the set of such $\mathbf{Q}^{\prime} s$ coincides precisely the group of rotation tensors, i.e.

$$
\mathbf{Q}^{-1}=\mathbf{Q}^{t} ; \quad \operatorname{det} \mathbf{Q}=1
$$

Consider an associated one-parameter family of deformation gradients defined by

$$
\mathbf{F}(u)=\mathbf{Q}(u) \mathbf{F}_{0}, \quad \text { with } \quad \mathbf{F}_{0}=\mathbf{F}(0)
$$

This is a rotation superposed on a deformation with gradient $\mathbf{F}_{0}$. Then,

$$
\dot{\mathbf{F}}=\dot{\mathbf{Q}} \mathbf{F}_{0}=\dot{\mathbf{Q}} \mathbf{Q}^{t} \mathbf{F}=\mathbf{\Omega F},
$$

and for this family of deformations we find, using (17) and the chain rule, that

$$
\dot{W}=W_{\mathbf{F}} \cdot \dot{\mathbf{F}}=0
$$

so that $W(\mathbf{F}(u))$ is independent of $u$, i.e.

$$
W\left(\mathbf{Q F}_{0}\right)=W\left(\mathbf{F}_{0}\right)
$$

in which the rotation $\mathbf{Q}$ is arbitrary.

A necessary condition follows on identifying $\mathbf{Q}$ with the transpose of the rotation factor $\mathbf{R}_{0}$ in the polar decomposition

$$
\mathbf{F}=\mathbf{R U}
$$

of the deformation gradient, where $\mathbf{U}$ is the positive definite, symmetric right-stretch tensor. This yields the conclusion that $W$ is determined by the stretch, i.e. $W\left(\mathbf{F}_{0}\right)=W\left(\mathbf{U}_{0}\right)$. However, this is inconvenient in practice because $\mathbf{U}$ is not easily obtained from $\mathbf{F}$. To circumvent this we note that there is a one-to-one relation between the right stretch and the symmetric Cauchy-Green deformation tensor $\mathbf{C}=\mathbf{U}^{2}=\mathbf{F}^{t} \mathbf{F}$; the former is the unique positive definite symmetric square root of the latter. We conclude that $\mathbf{U}$ is determined by $\mathbf{C}$ and hence (dropping subscripts in (23)) that $W(\mathbf{F})=\hat{W}(\mathbf{C})$ for some function $\hat{W}$. In turn, the Cauchy-Green tensor stands in one-to-one relation to the symmetric Lagrange strain

$$
\boldsymbol{\epsilon}=\frac{1}{2}\left(\mathbf{F}^{t} \mathbf{F}-\mathbf{I}\right)
$$

and so we may write $W(\mathbf{F})=\bar{W}(\boldsymbol{\epsilon})$ for some function $\bar{W}$. An elementary application of the chain rule then furnishes

$$
W_{\mathbf{F}}=\mathbf{F} \bar{W}_{\epsilon},
$$

where, on the right-hand side, $\bar{W}_{\epsilon}$ is the symmetric tensor-valued gradient with respect to strain. Accordingly, the 2nd Piola-Kirchhoff stress $\mathbf{S}$, defined by

$$
\mathbf{P}=\mathbf{F S}
$$


is given by

$$
\mathbf{S}=\bar{W}_{\epsilon}
$$

Comparison of (6) and (27) yields $J \mathbf{T}=\mathbf{F} \mathbf{S F}^{t}$, and so the symmetry of $\mathbf{S}$, implied by (23), yields the symmetry of $\mathbf{T}$. It follows that (23) is both necessary and sufficient for the symmetry of the Cauchy stress.

We neglect body forces, and thus reduce (5) and (15) to the system

$$
\operatorname{Div}\left(W_{\mathbf{F}}\right)=\rho_{\kappa} \chi_{t t}
$$

for the determination of the motion $\chi(\mathbf{x}, t)$, in which $\rho_{\kappa}$ is an assigned constant if, as we assume, the material properties are uniform.

In this work we restrict attention to deformations for which the strong ellipticity condition is satisfied, i.e.

$$
\mathbf{a} \otimes \mathbf{b} \cdot W_{\mathbf{F F}}[\mathbf{a} \otimes \mathbf{b}]>0 \quad \text { for all } \quad \mathbf{a} \otimes \mathbf{b} \neq \mathbf{0} .
$$

\section{Leading order model for a thin, nonlinearly elastic film in the long-wave limit}

We seek equations of motion for a thin film bonded to a substrate that are valid to leading order in the film thickness, presumed to be small against the length scale afforded by the wavelength of a propagating surface wave. Thus the model to be derived and studied is valid in the long-wave limit.

Consider a planar film of thickness $h$, bonded to an elastic half space. The interface between film and substrate, denoted by $\Omega$, is an unbounded plane with unit normal $\mathbf{k}$ directed away from the substrate. It proves advantageous to decompose three-dimensional position $\mathbf{x}$ in the film in terms of position $\mathbf{r}$ to a projected point on $\Omega$ and a linear coordinate $\varsigma$ in the direction of the normal. Thus,

$$
\mathbf{x}=\mathbf{r}+\varsigma \mathbf{k}, \quad \text { with } \quad \mathbf{r} \in \Omega \quad \text { and } \quad \varsigma \in[0, h]
$$

The motion of the film may then be regarded as a function of $\mathbf{r}$ and $\varsigma$, i.e. $\mathbf{y}=\boldsymbol{\chi}(\mathbf{x}, t)=\hat{\boldsymbol{\chi}}(\mathbf{r}, \varsigma, t)$; we also write $\mathbf{F}(\mathbf{x}, t)=\hat{\mathbf{F}}(\mathbf{r}, \varsigma, t)$. It then follows from the definition of the gradient that

$$
(\nabla \mathbf{y}) d \mathbf{r}+\mathbf{y}^{\prime} d \varsigma=d \mathbf{y}=\hat{\mathbf{F}} d \mathbf{x}=\hat{\mathbf{F}} \mathbf{1} d \mathbf{r}+\hat{\mathbf{F}} \mathbf{k} \otimes \mathbf{k} d \varsigma
$$

where $(\cdot)^{\prime}=\partial(\cdot) / \partial \varsigma, \nabla(\cdot)$ is the (two-dimensional) gradient with respect to $\mathbf{r}$ and

$$
\mathbf{1}=\mathbf{I}-\mathbf{k} \otimes \mathbf{k}
$$

is the projection onto the plane $\Omega$. This yields

$$
\nabla \mathbf{y}=\hat{\mathbf{F}} \mathbf{1} \quad \text { and } \quad \mathbf{y}^{\prime}=\hat{\mathbf{F}} \mathbf{k}
$$

Using a similar notation for the Piola stress, we write $\mathbf{P}=\hat{\mathbf{P}} \mathbf{1}+\hat{\mathbf{P}} \mathbf{k} \otimes \mathbf{k}$ and find that

$$
\operatorname{Div} \mathbf{P}=\operatorname{div}(\hat{\mathbf{P}} \mathbf{1})+\hat{\mathbf{P}}^{\prime} \mathbf{k}
$$


where, in contrast to its use in (2), div is now the (two-dimensional) referential divergence operator on $\Omega$. Thus (5) may be recast in the convenient form

$$
\operatorname{div}(\hat{\mathbf{P}} \mathbf{1})+\hat{\mathbf{P}}^{\prime} \mathbf{k}=\rho_{\kappa} \hat{\chi}_{t t} .
$$

We seek a two-dimensional model of the thin film, in terms of differential operators defined entirely on $\Omega$. To effect the dimension reduction, we adopt the weak form of the equations of motion in which the film thickness is made explicit. We then estimate this for small thickness and extract the leading-order local equations.

To this end let $\mathbf{y}(\mathbf{x}, t, \mu)$ be a one-parameter $(\mu)$ family of motions, let the actual motion $\mathbf{y}=\boldsymbol{\chi}(\mathbf{x}, t)$ be its value at $\mu=0$, and let

$$
\dot{\mathbf{y}}=\frac{\partial}{\partial \mu} \mathbf{y}(\mathbf{x}, t, \mu)_{\mid \mu=0} .
$$

Then the weak form of (5), holding in an arbitrary subvolume $\pi$ of the film, is

$$
\int_{\pi} \mathbf{P} \cdot \dot{\mathbf{F}} d V=\int_{\partial \pi} \mathbf{P N} \cdot \dot{\mathbf{y}} d A-\int_{\pi} \rho_{\kappa} \dot{\mathbf{y}} \cdot \mathbf{y}_{t t} d V
$$

in which

$$
\dot{\mathbf{F}}=\nabla \dot{\mathbf{y}}+\dot{\mathbf{y}}^{\prime} \otimes \mathbf{k}
$$

where $\dot{\mathbf{y}}^{\prime}=\left(\mathbf{y}^{\prime}\right)^{\cdot}=(\dot{\mathbf{y}})^{\prime}$. We remark that, on the film/substrate interface $\Omega$, the deformation gradient is

$$
\mathbf{F}_{0}=\nabla \mathbf{y}_{0}+\mathbf{d} \otimes \mathbf{k}
$$

where, here and henceforth, the notation $(\cdot)_{0}$ stands for the restriction $(\cdot)_{\mid \Omega}$ of a variable defined in the film. This is the interior limit of the considered quantity as $\varsigma \rightarrow 0$. In particular,

$$
\mathbf{y}_{0}(\mathbf{r}, t)=\hat{\mathbf{y}}(\mathbf{r}, 0, t) \quad \text { and } \quad \mathbf{d}(\mathbf{r}, t)=\hat{\mathbf{y}}^{\prime}(\mathbf{r}, 0, t)
$$

and these are independent vector fields on $\Omega$.

Proceeding from (38) and (39), we have

$$
\int_{\pi} \mathbf{P} \cdot \dot{\mathbf{F}} d V=\int_{\pi}\left(\mathbf{P} \mathbf{1} \cdot \nabla \dot{\mathbf{y}}+\mathbf{P k} \cdot \dot{\mathbf{y}}^{\prime}\right) d V=\int_{\omega} \int_{0}^{h}\left(\mathbf{P} \mathbf{1} \cdot \nabla \dot{\mathbf{y}}+\mathbf{P k} \cdot \dot{\mathbf{y}}^{\prime}\right) d \varsigma d A
$$

where $\omega \subset \Omega$ is an arbitrary part of $\Omega$ and we have selected $\pi=\omega \times[0, h]$. For an arbitrary function $g(\mathbf{r}, \varsigma)$ we use the Taylor expansion

$$
\int_{0}^{h} g d \varsigma:=I(\mathbf{r}, h)=h I^{\prime}(\mathbf{r}, 0)+o(h), \quad \text { with } \quad I^{\prime}(\mathbf{r}, h)=g(\mathbf{r}, h),
$$

to derive the estimate

$$
I(\mathbf{r}, h)=h g_{0}+o(h)
$$

and thus estimate (42) as

$$
\int_{\pi} \mathbf{P} \cdot \dot{\mathbf{F}} d V=h \int_{\omega}\left(\mathbf{P}_{0} \mathbf{1} \cdot \nabla \dot{\mathbf{y}}_{0}+\mathbf{P}_{0} \mathbf{k} \cdot \dot{\mathbf{d}}\right) d A+o(h) .
$$

Similarly,

$$
\int_{\pi} \rho_{\kappa} \dot{\mathbf{y}} \cdot \mathbf{y}_{t t} d V=h \int_{\omega} \rho_{\kappa} \dot{\mathbf{y}}_{0} \cdot \mathbf{y}_{0 t t} d A+o(h)
$$


The remaining integral in (38) may be decomposed as

$$
\int_{\partial \pi} \mathbf{P N} \cdot \dot{\mathbf{y}} d A=\int_{\partial \omega} \int_{0}^{h} \mathbf{P} 1 \nu \cdot \dot{\mathbf{y}} d \varsigma d S+\int_{\omega^{+}} \mathbf{P}^{+} \mathbf{k} \cdot \dot{\mathbf{y}}^{+} d A-\int_{\omega} \mathbf{P}_{0} \mathbf{k} \cdot \dot{\mathbf{y}}_{0} d A
$$

where $\omega^{+}$is the upper surface of the film at a distance $h$ from $\omega, \mathbf{k}$ and $-\mathbf{k}$ are the exterior unit normals to the film at $\omega^{+}$and $\omega$, the superscript + is used to denote the values of variables at $\varsigma=h$, and $\boldsymbol{\nu}$ is the external unit normal to the cylindrical generating surface $\partial \omega \times[0, h]$.

Traction continuity at the film/substrate interface $\Omega$ implies that

$$
\mathbf{P}_{0} \mathbf{k}=\mathbf{P}_{s} \mathbf{k}
$$

where $\mathbf{P}_{s}$ is the limiting value of the substrate stress on $\Omega$. Assuming the upper surface of the film to be traction free, i.e. $\mathbf{P}^{+} \mathbf{k}=\mathbf{0}$, and using the rule (44), we then have

$$
\int_{\partial \pi} \mathbf{P N} \cdot \dot{\mathbf{y}} d A=h \int_{\partial \omega} \mathbf{P}_{0} \mathbf{1} \nu \cdot \dot{\mathbf{y}}_{0} d S-\int_{\omega} \mathbf{P}_{s} \mathbf{k} \cdot \dot{\mathbf{y}}_{0} d A+o(h) .
$$

Substituting this, together with (45) and (46), into (38), we conclude that

$$
\int_{\omega} \mathbf{P}_{s} \mathbf{k} \cdot \dot{\mathbf{y}}_{0} d A=O(h)
$$

and hence, from the arbitrariness of $\omega$ and $\dot{\mathbf{y}}_{0}$, that $\mathbf{P}_{s} \mathbf{k}=O(h)$, i.e.

$$
\mathbf{P}_{s} \mathbf{k}=h \mathbf{l}+o(h)
$$

in which $\mathbf{l}(\mathbf{r}, t)$ is independent of $h$. Substituting back into the balance (38), dividing by $h$ and passing to the limit then yields

$$
\int_{\omega}\left[\mathbf{P}_{0} \mathbf{1} \cdot \nabla \dot{\mathbf{y}}_{0}+\mathbf{P}_{0} \mathbf{k} \cdot \dot{\mathbf{d}}+\dot{\mathbf{y}}_{0} \cdot\left(\rho_{\kappa} \mathbf{y}_{0 t t}+\mathbf{l}\right)\right] d A=\int_{\partial \omega} \mathbf{P}_{0} \mathbf{1} \nu \cdot \dot{\mathbf{y}}_{0} d S
$$

Applying Green's theorem in the form

$$
\int_{\partial \omega} \mathbf{P}_{0} \mathbf{1} \boldsymbol{\nu} \cdot \dot{\mathbf{y}}_{0} d S=\int_{\partial \omega} \boldsymbol{\nu} \cdot\left(\mathbf{P}_{\mathbf{0}} \mathbf{1}\right)^{t} \dot{\mathbf{y}}_{0} d S=\int_{\omega} \operatorname{div}\left[\left(\mathbf{P}_{\mathbf{0}} \mathbf{1}\right)^{t} \dot{\mathbf{y}}_{0}\right] d A=\int_{\omega}\left[\dot{\mathbf{y}}_{0} \cdot \operatorname{div}\left(\mathbf{P}_{0} \mathbf{1}\right)+\mathbf{P}_{0} \mathbf{1} \cdot \nabla \dot{\mathbf{y}}_{0}\right] d A
$$

we reduce (52) to

$$
\int_{\omega}\left\{\mathbf{P}_{0} \mathbf{k} \cdot \dot{\mathbf{d}}-\dot{\mathbf{y}}_{0} \cdot\left[\operatorname{div}\left(\mathbf{P}_{0} \mathbf{1}\right)-\mathbf{l}-\rho_{\kappa} \mathbf{y}_{0 t t}\right]\right\} d A=0
$$

and then invoke the arbitrariness of $\omega$ and the independence of $\dot{\mathbf{y}}_{0}$ and $\dot{\mathbf{d}}$ to extract the local equations

$$
\mathbf{P}_{0} \mathbf{k}=\mathbf{0} \quad \text { and } \quad \operatorname{div}\left(\mathbf{P}_{0} \mathbf{1}\right)-\mathbf{l}=\rho_{\kappa} \mathbf{y}_{0 t t} \quad \text { on } \quad \Omega .
$$

On multiplying the second of these by $h$, using (51) and neglecting terms of order $o(h)$ we obtain

$$
h \operatorname{div}\left(\mathbf{P}_{0} \mathbf{1}\right)-\mathbf{P}_{s} \mathbf{k}=h \rho_{\kappa} \mathbf{y}_{0 t t} .
$$

This is the rigorous leading-order (in $h$ ) boundary condition for the substrate at the film/substrate interface $\Omega$, reducing to the usual traction-free condition $\mathbf{P}_{s} \mathbf{k}=\mathbf{0}$ in the absence of the film $(h=0)$. It is also seen to furnish the leading order equation of motion for the film/substrate interface. Similarly, the first of (55) is the leading-order approximation of the traction continuity condition (48), with (51). This condition implies that, to leading order, the film is in a state of plane stress. 
In these equations the stress $\mathbf{P}_{0}$ is given by

$$
\mathbf{P}_{0}=W_{\mathbf{F}}\left(\nabla \mathbf{y}_{0}+\mathbf{d} \otimes \mathbf{k}\right)
$$

and so (55) may be regarded as a system for the independent fields $\mathbf{y}_{0}$ and $\mathbf{d}$ on $\Omega$.

It happens that in the presence of strong ellipticity (55) $)_{1}$ may be solved for $\mathbf{d}$ in terms of $\nabla \mathbf{y}_{0}$. To see this we fix $\mathbf{y}_{0}$ and define $G(\mathbf{d})=W\left(\nabla \mathbf{y}_{0}+\mathbf{d} \otimes \mathbf{k}\right)$. Let $\sigma(u)=G(\mathbf{d}(u))$, for some parameter $u$. Then,

$$
\dot{\sigma}(u)=W_{\mathbf{F}}\left(\mathbf{F}_{0}\right) \cdot \dot{\mathbf{d}} \otimes \mathbf{k}=\dot{\mathbf{d}} \cdot \mathbf{P}_{0} \mathbf{k}, \quad \text { and thus } \quad G_{\mathbf{d}}=\mathbf{P}_{0} \mathbf{k} .
$$

Further,

$$
\ddot{\sigma}(u)=\ddot{\mathbf{d}} \cdot \mathbf{P}_{0} \mathbf{k}+\dot{\mathbf{d}} \otimes \mathbf{k} \cdot \mathcal{M}\left(\mathbf{F}_{0}\right)[\dot{\mathbf{d}} \otimes \mathbf{k}]
$$

where

$$
\mathcal{M}=W_{\mathbf{F F}}
$$

and so

$$
G_{\mathbf{d d}}=\mathbf{A}\left(\mathbf{F}_{0}\right)
$$

where $\mathbf{A}$ is the acoustic tensor defined by

$$
\mathbf{A v}=\left\{\mathcal{M}\left(\mathbf{F}_{0}\right)[\mathbf{v} \otimes \mathbf{k}]\right\} \mathbf{k}
$$

for all vectors $\mathbf{v}$. In terms of components,

$$
A_{i j}=\partial^{2} W / \partial F_{i 3} \partial F_{j 3}
$$

having made the identification $\mathbf{e}_{3}=\mathbf{k}$. It follows from (30) that $G_{\mathbf{d d}}$ is positive definite and hence, from the implicit function theorem, that $(55)_{1}$ (i.e. $\left.G_{\mathbf{d}}=\mathbf{0}\right)$, has a unique solution $\mathbf{d}=\overline{\mathbf{d}}\left(\nabla \mathbf{y}_{0}\right)$, say, as claimed. Further, the foregoing implies that this solution minimizes the energy $W\left(\nabla \mathbf{y}_{0}+\mathbf{d} \otimes \mathbf{k}\right)$ with respect to $\mathbf{d}$.

In this work it is convenient to work with strain-dependent moduli. To elaborate, consider a oneparameter family $\mathbf{F}(u)$ of deformations and let $\boldsymbol{\epsilon}(u)$ be the associated strain. Using the connection (27) we then have

$$
\mathcal{M}[\dot{\mathbf{F}}]=\dot{\mathbf{P}}=\dot{\mathbf{F}} \mathbf{S}+\mathbf{F} \mathcal{C}[\dot{\boldsymbol{\epsilon}}], \quad \text { with } \quad \dot{\boldsymbol{\epsilon}}=\frac{1}{2}\left(\dot{\mathbf{F}}^{t} \mathbf{F}+\mathbf{F}^{t} \dot{\mathbf{F}}\right),
$$

where the superposed dots are derivatives with respect to the parameter, and

$$
\mathcal{C}=\bar{W}_{\epsilon \epsilon}
$$

are the strain-dependent moduli. We note that this possesses both major and minor symmetries whereas $\mathcal{M}$ possesses only major symmetry. Accordingly,

$$
\mathcal{M}[\mathbf{B}]=\mathbf{B S}+\mathbf{F C}\left[\operatorname{Sym}\left(\mathbf{B}^{t} \mathbf{F}\right)\right], \quad \text { for all tensors } \mathbf{B},
$$

and the strong-ellipticity condition (30) is thus seen to be equivalent to

$$
(\mathbf{b} \cdot \mathbf{S b})|\mathbf{a}|^{2}+\mathbf{F}^{t} \mathbf{a} \otimes \mathbf{b} \cdot \mathcal{C}\left[\mathbf{F}^{t} \mathbf{a} \otimes \mathbf{b}\right]>0
$$


For our purposes the relevant restriction pertains to small perturbations of the reference configuration associated with $\mathbf{F}=\mathbf{I}$. This is

$$
\left(\mathbf{b} \cdot \mathbf{S}_{R} \mathbf{b}\right)|\mathbf{a}|^{2}+\mathbf{a} \otimes \mathbf{b} \cdot \mathcal{C}_{R}[\mathbf{a} \otimes \mathbf{b}]>0
$$

where $\mathbf{S}_{R}$ is the residual stress in that configuration and $\mathcal{C}_{R}$ is the associated tensor of elastic moduli. There are equal respectively to the values of $\mathbf{S}$ and $\mathcal{C}$ at $\boldsymbol{\epsilon}=\mathbf{0}$.

\section{Linearization}

To linearize the equations we introduce the displacement field

$$
\mathbf{u}(\mathbf{x}, t)=\chi(\mathbf{x}, t)-\mathbf{x}
$$

and assume that $\sup _{\mathbf{x} \in \kappa}|\mathbf{H}(\mathbf{x}, t)| \ll 1$, where

$$
\mathbf{H}=\operatorname{Grad} \mathbf{u}
$$

is the displacement gradient. From (1) and (69) we have the exact expressions

$$
\mathbf{F}=\mathbf{I}+\mathbf{H} \quad \text { and } \quad \boldsymbol{\epsilon}=\frac{1}{2}\left(\mathbf{H}+\mathbf{H}^{t}+\mathbf{H}^{t} \mathbf{H}\right),
$$

and our assumptions imply that $\sup _{\mathbf{x} \in \kappa}|\boldsymbol{\epsilon}(\mathbf{x}, t)| \ll 1$. Accordingly, the estimate

$$
\bar{W}_{\boldsymbol{\epsilon}}=\bar{W}_{\boldsymbol{\epsilon}}(\mathbf{0})+\bar{W}_{\boldsymbol{\epsilon} \boldsymbol{\epsilon}}(\mathbf{0})[\operatorname{Sym} \mathbf{H}]+o(|\mathbf{H}|)
$$

is appropriate, in which the coefficients are the values of the stress and moduli at $\boldsymbol{\epsilon}=\mathbf{0}$, and furnishes the linearized stress-deformation relations

$$
\mathbf{S} \simeq \mathbf{S}_{R}+\mathcal{C}_{R}[\mathbf{H}] \quad \text { and } \quad \mathbf{P}=(\mathbf{I}+\mathbf{H}) \mathbf{S} \simeq \mathbf{S}_{R}+\mathbf{H S}_{R}+\mathcal{C}_{R}[\mathbf{H}]
$$

The residual stress and associated moduli are necessarily uniform if the material is homogeneous. Assuming the body to be in equilibrium without tractions prior to undergoing the small displacement, we have

$$
\operatorname{Div} \mathbf{S}_{R}=\mathbf{0} \quad \text { in } \quad \kappa \text { and } \mathbf{S}_{R} \mathbf{N}=\mathbf{0} \text { on } \partial \kappa_{t},
$$

the first of which is then identically satisfied.

The thin-film condition (55) 1 may be expressed in the form

$$
\mathbf{S}_{R} \mathbf{k}+\mathbf{H}_{0} \mathbf{S}_{R} \mathbf{k}+\left(\mathcal{C}_{R}\left[\mathbf{H}_{0}\right]\right) \mathbf{k}=\mathbf{0},
$$

and as this purports to hold for all deformations it follows that

$$
\mathbf{S}_{R} \mathbf{k}=\mathbf{0} \quad \text { and } \quad\left(\mathcal{C}_{R}\left[\mathbf{H}_{0}\right]\right) \mathbf{k}=\mathbf{0}
$$

the first of these implying that $\mathbf{S}_{R}$ is a (symmetric) two-tensor of the form $\mathbf{S}_{R}=S_{R \alpha \beta} \mathbf{e}_{\alpha} \otimes \mathbf{e}_{\beta}$, where $\left\{\mathbf{e}_{\alpha}\right\}$ is an orthonormal basis in the plane $\Omega$. To investigate the consequences of the second restriction we write $\mathbf{H}_{0}$, the restriction of the film displacement gradient to $\Omega$, in the form (40), obtaining

$$
\mathbf{H}_{0}=\nabla \mathbf{u}_{0}+\mathbf{u}_{0}^{\prime} \otimes \mathbf{k}
$$


where $\mathbf{u}_{0}(\mathbf{r}, t)$ is the displacement of the film/substrate interface and $\mathbf{u}_{0}^{\prime}$ is the restriction to $\Omega$ of the through-thickness derivative $\mathbf{u}^{\prime}$ of the displacement field in the film. The stated restriction may then be written in the form

$$
\left(\mathcal{C}_{R}\left[\nabla \mathbf{u}_{0}\right]\right) \mathbf{k}+\mathbf{A}_{R} \mathbf{u}_{0}^{\prime}=\mathbf{0}
$$

where $\mathbf{A}_{R}$ is the relevant acoustic tensor, defined for all $\mathbf{v}$ by

$$
\mathbf{A}_{R} \mathbf{v}=\left(\mathcal{C}_{R}[\mathbf{v} \otimes \mathbf{k}]\right) \mathbf{k}
$$

and which is positive definite by (68) and $(76)_{1}$. Consequently,

$$
\mathbf{u}_{0}^{\prime}=\mathbf{a}\left(\nabla \mathbf{u}_{0}\right), \quad \text { with } \quad \mathbf{a}(\cdot)=-\mathbf{A}_{R}^{-1}\left(\mathcal{C}_{R}[\cdot]\right) \mathbf{k},
$$

implying that $\mathbf{H}_{0}$ is determined entirely by the interfacial displacement.

The interfacial equation of motion (56) requires an expression for $\mathbf{P}_{0} \mathbf{1}$, which, on making use of $(73)_{2}$, is given to linear order by

$$
\mathbf{P}_{0} \mathbf{1}=\mathbf{S}_{R}+\mathbf{H}_{0} \mathbf{S}_{R}+\left(\mathcal{C}_{R}\left[\mathbf{H}_{0}\right]\right) \mathbf{1}, \quad \text { with } \quad \mathbf{H}_{0}=\nabla \mathbf{u}_{0}+\mathbf{a}\left(\nabla \mathbf{u}_{0}\right) \otimes \mathbf{k}
$$

This is used in (56) in the form

$$
\mathbf{P}_{s} \mathbf{k}=h\left[\operatorname{div}\left(\mathbf{P}_{0} \mathbf{1}\right)-\rho_{\kappa} \mathbf{u}_{0 t t}\right]
$$

in which $\mathbf{P}_{s}$ is the restriction to $\Omega$ of the substrate stress, assumed here to be given constitutively by $\mathbf{P}_{s}=\boldsymbol{\sigma}_{0}$, where

$$
\boldsymbol{\sigma}=\mathcal{E}[\mathbf{G}] ; \quad \mathbf{G}=\operatorname{Grad} \mathbf{w}
$$

in which $\mathcal{E}$ is the substrate elasticity tensor (possessing major and minor symmetries) and $\mathbf{w}(\mathbf{x}, t)$ is the substrate displacement field. This satisfies $\mathbf{w}_{0}=\mathbf{u}_{0}$ in a perfectly bonded film-substrate system but of course $\mathbf{w}_{0}^{\prime} \neq \mathbf{u}_{0}^{\prime}$, in general. This expression for the stress presumes the substrate to be free of residual stress. Generalizations are of course possible, but the present simplification suffices for our purposes.

To make the equations explicit it is convenient to decompose the interfacial displacement into tangential and normal parts, i.e.

$$
\mathbf{u}_{0}=\mathbf{v}+w \mathbf{k}, \quad \text { where } \quad \mathbf{v}=\mathbf{1} \mathbf{u}_{0} \quad \text { and } \quad w=\mathbf{k} \cdot \mathbf{u}_{0}
$$

In the same way we write

$$
\mathbf{a}=\boldsymbol{\alpha}+a \mathbf{k}, \quad \text { where } \quad \boldsymbol{\alpha}=\mathbf{1} \mathbf{a} \text { and } \quad a=\mathbf{k} \cdot \mathbf{a} .
$$

These furnish

$$
\nabla \mathbf{u}_{0}=\nabla \mathbf{v}+\mathbf{k} \otimes \nabla w \quad \text { and } \quad \mathbf{a} \otimes \mathbf{k}=\boldsymbol{\alpha} \otimes \mathbf{k}+a \mathbf{k} \otimes \mathbf{k}
$$

and hence afford the representation

$$
\mathcal{C}_{R}\left[\mathbf{H}_{0}\right]=\left(\mathcal{C}_{i j \alpha \beta} v_{\alpha, \beta}+\mathcal{C}_{i j 3 \alpha} w_{, \alpha}+\mathcal{C}_{i j \beta 3} \alpha_{\beta}+a \mathcal{C}_{i j 33}\right) \mathbf{e}_{i} \otimes \mathbf{e}_{j},
$$

in which the subscript ${ }_{R}$ has been suppressed on the right-hand side. 
The material properties considered in this work exhibit reflection symmetry with respect to the unit normal $\mathbf{k}$, i.e.

$$
\bar{W}(\boldsymbol{\epsilon})=\bar{W}\left(\mathbf{Q} \epsilon \mathbf{Q}^{t}\right), \quad \text { with } \quad \mathbf{Q}=\mathbf{I}-2 \mathbf{k} \otimes \mathbf{k} .
$$

These have the property that all components $\mathcal{C}_{i j k l}$ of the elastic moduli having an odd number of subscripts equal to 3 vanish (Green and Zerna, 1968); hence the simplification

$$
\begin{aligned}
\mathcal{C}_{R}\left[\mathbf{H}_{0}\right]= & \mathcal{C}_{\lambda \mu \alpha \beta} v_{\alpha, \beta} \mathbf{e}_{\lambda} \otimes \mathbf{e}_{\mu}+a \mathcal{C}_{\alpha \beta 33} \mathbf{e}_{\alpha} \otimes \mathbf{e}_{\beta}+\left(\mathcal{C}_{33 \alpha \beta} v_{\alpha, \beta}+a \mathcal{C}_{3333}\right) \mathbf{k} \otimes \mathbf{k} \\
& +\mathcal{C}_{\alpha 3 \beta 3}\left(w_{, \beta}+\alpha_{\beta}\right)\left(\mathbf{e}_{\alpha} \otimes \mathbf{k}+\mathbf{k} \otimes \mathbf{e}_{\alpha}\right) .
\end{aligned}
$$

Using the definition (79) of the acoustic tensor we then obtain

$$
\mathbf{A}_{R} \mathbf{b}=\mathcal{C}_{\alpha 3 \beta 3} b_{\beta} \mathbf{e}_{\alpha}+C b_{3} \mathbf{k}, \text { where } C=\mathcal{C}_{3333},
$$

yielding

$$
\mathbf{b} \cdot \mathbf{A}_{R} \mathbf{b}=\mathcal{C}_{\alpha 3 \beta 3} b_{\alpha} b_{\beta}+C b_{3}^{2}
$$

The acoustic tensor is then positive definite as required if and only if $C>0$ and $\left(\mathcal{C}_{\alpha 3 \beta 3}\right)$ is positive definite. Further, (84) and (85) give

$$
\mathbf{A}_{R} \mathbf{a}=\mathcal{C}_{\alpha 3 \beta 3} \alpha_{\beta} \mathbf{e}_{\alpha}+C a \mathbf{k} \quad \text { and } \quad\left(\mathcal{C}_{R}\left[\nabla \mathbf{u}_{0}\right]\right) \mathbf{k}=\mathcal{C}_{33 \alpha \beta} v_{\alpha, \beta} \mathbf{k}+\mathcal{C}_{\alpha 3 \beta 3} w_{, \beta} \mathbf{e}_{\alpha}
$$

and it follows from (78) that

$$
\boldsymbol{\alpha}=-\nabla w, \quad a=-C^{-1} \mathcal{C}_{33 \alpha \beta} v_{\alpha, \beta} .
$$

The restriction $\mathbf{H}_{0}$ to $\Omega$ of the film displacement gradient is thus given by

$$
\mathbf{H}_{0}=\nabla \mathbf{v}+a \mathbf{k} \otimes \mathbf{k}+\mathbf{k} \otimes \nabla w-\nabla w \otimes \mathbf{k}
$$

and (89), together with the minor symmetry of $\mathcal{C}_{R}$, yields

$$
\mathcal{C}_{R}\left[\mathbf{H}_{0}\right]=\mathcal{D}[\nabla \mathbf{v}]=\mathcal{D}_{\lambda \mu \alpha \beta} v_{\alpha, \beta} \mathbf{e}_{\lambda} \otimes \mathbf{e}_{\mu}
$$

where

$$
\mathcal{D}_{\lambda \mu \alpha \beta}=\mathcal{C}_{\lambda \mu \alpha \beta}-C^{-1} \mathcal{C}_{33 \lambda \mu} \mathcal{C}_{33 \alpha \beta}
$$

are the plane-stress moduli. This in turn furnishes

$$
\mathbf{P}_{0} \mathbf{1}=\mathbf{S}_{R}+(\nabla \mathbf{v}) \mathbf{S}_{R}+\mathbf{k} \otimes\left(\mathbf{S}_{R} \nabla w\right)+\mathcal{D}[\nabla \mathbf{v}]
$$

and eq. (82) for the interfacial motion reduces to

$$
\boldsymbol{\sigma}_{0} \mathbf{k}=h\left[\operatorname{div}\left\{(\nabla \mathbf{v}) \mathbf{S}_{R}+\mathcal{D}[\nabla \mathbf{v}]\right\}+\mathbf{k} \operatorname{div}\left(\mathbf{S}_{R} \nabla w\right)-\rho_{\kappa} \mathbf{v}_{t t}-\rho_{\kappa} w_{t t} \mathbf{k}\right]
$$

The substrate displacement is described by the classical equation of motion

$$
\operatorname{Div} \boldsymbol{\sigma}=\rho_{s} \mathbf{w}_{t t},
$$

where $\rho_{s}$ is the substrate mass density. 


\section{Surface waves: Love modes in hexagonal and cubic crystal films}

We are interested in localized surface waves of the form

$$
\mathbf{w}(\mathbf{x}, t)=F(\mathbf{x}, t) \mathbf{d}
$$

in which $\mathbf{d}$ is the fixed polarization vector, $\mathbf{x}$ is decomposed as in (31) in which $\varsigma<0$ for the substrate, and

$$
F(\mathbf{x}, t)=\exp (\eta k \varsigma) \exp [i k(\mathbf{n} \cdot \mathbf{r}-c t)]
$$

in which $\eta$ is a constant - assumed positive to ensure decay with depth into the substrate, $\mathbf{n} \in \Omega$ is the propagation direction, $c$ is the wave speed, and $k$ is the wave number. The induced interfacial displacement is

$$
\mathbf{u}_{0}=\mathbf{w}_{0}=G \mathbf{d}, \quad \text { where } \quad G=F_{0}=\exp [i k(\mathbf{n} \cdot \mathbf{r}-c t)]
$$

The gradient of the interfacial displacement is

$$
\nabla \mathbf{u}_{0}=\mathbf{d} \otimes \nabla G=i k G \mathbf{d} \otimes \mathbf{n}
$$

It proves convenient to decompose the polarization in the form (84), i.e.

$$
\mathbf{d}=\boldsymbol{\delta}+d \mathbf{k} ; \quad \delta=\mathbf{1 d}, \quad d=\mathbf{k} \cdot \mathbf{d} .
$$

Then,

$$
\nabla \mathbf{v}=i k G \boldsymbol{\delta} \otimes \mathbf{n} \quad \text { and } \quad \nabla w=i k G d \mathbf{n} .
$$

Using these results with $\nabla \nabla G=-k^{2} G \mathbf{n} \otimes \mathbf{n}$ and $\mathbf{u}_{0 t t}=-k^{2} c^{2} G \mathbf{d}$, after some effort we reduce the bracketed term on the right-hand side of (82) to

$$
\operatorname{div}\left(\mathbf{P}_{0} \mathbf{1}\right)-\rho_{\kappa} \mathbf{u}_{0 t t}=G k^{2}\left\{\left(\rho_{\kappa} c^{2}-\mathbf{n} \cdot \mathbf{S}_{R} \mathbf{n}\right) \mathbf{d}-\mathcal{A} \boldsymbol{\delta}\right\}
$$

where

$$
\mathcal{A}=\mathcal{D}_{\beta \alpha \lambda \mu} n_{\alpha} n_{\mu} \mathbf{e}_{\beta} \otimes \mathbf{e}_{\lambda}
$$

is the (symmetric) plane-stress acoustic tensor associated with the propagation direction.

The induced stress in the substrate is given by (83) with

$$
\mathbf{G}=\mathbf{d} \otimes G r a d F, \quad \text { where } \quad G r a d F=k F(i \mathbf{n}+\eta \mathbf{k}),
$$

yielding

$$
\mathbf{G}=k F(i \mathbf{d} \otimes \mathbf{n}+\eta \mathbf{d} \otimes \mathbf{k})
$$

and hence

$$
\boldsymbol{\sigma}=k F \mathbf{B}, \quad \text { where } \quad \mathbf{B}=i \mathcal{E}[\mathbf{d} \otimes \mathbf{n}]+\eta \mathcal{E}[\mathbf{d} \otimes \mathbf{k}] .
$$

Using this in the interfacial equation of motion (82) leads to the algebraic problem

$$
\mathbf{B k}=\epsilon\left\{\left(\rho_{\kappa} c^{2}-\mathbf{n} \cdot \mathbf{S}_{R} \mathbf{n}\right) \mathbf{d}-\mathcal{A} \boldsymbol{\delta}\right\}
$$


where

$$
\epsilon=h k \ll 1
$$

is the dimensionless film thickness.

\subsection{Substrate motion}

From (100) and (101) we have $\mathbf{w}_{t t}=-k^{2} c^{2} F \mathbf{d}$ and

$$
D i v \boldsymbol{\sigma}=k \mathbf{B}(G r a d F)=k^{2} F \mathbf{B}(i \mathbf{n}+\eta \mathbf{k})
$$

which reduce the substrate equation of motion (cf. (99)) to the algebraic problem

$$
-(\mathcal{E}[\mathbf{d} \otimes \mathbf{n}]) \mathbf{n}+i \eta\{(\mathcal{E}[\mathbf{d} \otimes \mathbf{k}]) \mathbf{n}+(\mathcal{E}[\mathbf{d} \otimes \mathbf{n}]) \mathbf{k}\}+\eta^{2}(\mathcal{E}[\mathbf{d} \otimes \mathbf{k}]) \mathbf{k}=-\rho_{s} c^{2} \mathbf{d}
$$

In isotropic substrates, to which attention is restricted in this work,

$$
\mathcal{E}[\mathbf{G}]=\lambda_{s}(\operatorname{tr} \mathbf{G}) \mathbf{I}+2 \mu_{s} \mathbf{G},
$$

where $\lambda_{s}$ and $\mu_{s}$ are the substrate Lamé moduli, assumed here to satisfy the usual strong ellipticity conditions $\lambda_{s}+2 \mu_{s}>0$ and $\mu_{s}>0$. In this case straightforward calculation reduces (114) to the system

$$
\begin{aligned}
\mu_{s}(i d \mathbf{n}+\eta \boldsymbol{\delta}) & =\epsilon\left[\left(\rho_{\kappa} c^{2}-\mathbf{n} \cdot \mathbf{S}_{R} \mathbf{n}\right) \boldsymbol{\delta}-\mathcal{A} \boldsymbol{\delta}\right] \\
i \lambda_{s}(\mathbf{n} \cdot \boldsymbol{\delta})+\eta\left(\lambda_{s}+2 \mu_{s}\right) d & =\epsilon\left(\rho_{\kappa} c^{2}-\mathbf{n} \cdot \mathbf{S}_{R} \mathbf{n}\right) d
\end{aligned}
$$

Further,

$$
\begin{aligned}
(\mathcal{E}[\mathbf{d} \otimes \mathbf{k}]) \mathbf{k} & =\left(\lambda_{s}+2 \mu_{s}\right) d \mathbf{k}+\mu_{s} \boldsymbol{\delta}, \quad(\mathcal{E}[\mathbf{d} \otimes \mathbf{n}]) \mathbf{k}=\lambda_{s}(\mathbf{n} \cdot \boldsymbol{\delta}) \mathbf{k}+\mu_{s} d \mathbf{n}, \\
(\mathcal{E}[\mathbf{d} \otimes \mathbf{n}]) \mathbf{n} & =\left(\lambda_{s}+\mu_{s}\right)(\mathbf{n} \cdot \boldsymbol{\delta}) \mathbf{n}+\mu_{s} \mathbf{d}, \quad(\mathcal{E}[\mathbf{d} \otimes \mathbf{k}]) \mathbf{n}=\lambda_{s} d \mathbf{n}+\mu_{s}(\mathbf{n} \cdot \boldsymbol{\delta}) \mathbf{k}
\end{aligned}
$$

Love waves are polarized in the plane $\Omega$. Accordingly, $d=0$ and $(116)_{2}$ requires that

$$
\mathbf{n} \cdot \boldsymbol{\delta}=0
$$

Eqs. (117) simplify dramatically to

$$
(\mathcal{E}[\mathbf{d} \otimes \mathbf{k}]) \mathbf{k}=\mu_{s} \boldsymbol{\delta}, \quad(\mathcal{E}[\mathbf{d} \otimes \mathbf{n}]) \mathbf{k}=\mathbf{0}, \quad(\mathcal{E}[\mathbf{d} \otimes \mathbf{n}]) \mathbf{n}=\mu_{s} \boldsymbol{\delta} \quad \text { and } \quad(\mathcal{E}[\mathbf{d} \otimes \mathbf{k}]) \mathbf{n}=\mathbf{0},
$$

and (99) delivers

$$
\eta=\sqrt{1-s^{2}}, \quad \text { where } \quad s=c / c_{s}
$$

and $c_{s}=\sqrt{\mu_{s} / \rho_{s}}$ is the shear-wave speed in the substrate. Finally, $(116)_{1}$ reduces to the propagation condition

$$
\mu_{s} \eta \boldsymbol{\delta}=\epsilon\left[\left(\rho_{\kappa} c^{2}-\mathbf{n} \cdot \mathbf{S}_{R} \mathbf{n}\right) \boldsymbol{\delta}-\mathcal{A} \boldsymbol{\delta}\right]
$$

which requires that $\boldsymbol{\delta}$ be an eigenvector of the acoustic tensor $\mathcal{A}$. The restriction (118) and the symmetry of the acoustic tensor imply that the propagation direction $\mathbf{n}$ is then also an eigenvector. 
5.2 Hexagonal (fibre) symmetry

In this subsection explicit dispersion relations are derived for films having hexagonal symmetry. In the linear theory, the associated constitutive equations are of precisely the same form as those for materials exhibiting transverse isotropy, or fibre symmetry. Accordingly, the results derived are applicable to both fibre-reinforced film materials or hexagonal crystalline materials.

In particular, the components of $\mathcal{C}$ relative to an orthonormal basis $\left\{\mathbf{e}_{i}\right\}$ are (see Spencer, 1984)

$$
\begin{aligned}
\mathcal{C}_{i j k l}= & \lambda \delta_{i j} \delta_{k l}+\mu_{T}\left(\delta_{i k} \delta_{j l}+\delta_{i l} \delta_{j k}\right)+\alpha\left(\delta_{i j} m_{k} m_{l}+m_{i} m_{j} \delta_{k l}\right) \\
& +\left(\mu_{L}-\mu_{T}\right)\left(m_{i} m_{k} \delta_{j l}+m_{i} m_{l} \delta_{j k}+m_{j} m_{k} \delta_{i l}+m_{j} m_{l} \delta_{i k}\right)+\beta m_{i} m_{j} m_{k} m_{l},
\end{aligned}
$$

where $\delta_{i j}$ is the Kronecker delta; $\alpha, \beta, \lambda, \mu_{T}$ and $\mu_{L}$ are material constants; and the unit vector $\mathbf{m}$, with components $m_{i}$, is the fiber axis, assumed here to be uniform. Spencer (1984) shows that $\mu_{T}$ is the shear modulus for shearing in planes transverse to $\mathbf{m}$, whereas $\mu_{L}$ is the shear modulus for shearing parallel to $\mathbf{m}$. The remaining material constants in (122) may be interpreted in terms of extensional moduli and Poisson ratios (Spencer, 1984).

The general form of the residual stress may be derived by enumerating the strain invariants for transverse isotropy that are linear in the (infinitesimal) strain. These are (Spencer, 1984) I $\cdot \boldsymbol{\epsilon}$ and $\mathbf{m} \otimes \mathbf{m} \cdot \boldsymbol{\epsilon}$. Comparison with the leading term in (72) then furnishes

$$
\mathbf{S}_{R}=S_{T}(\mathbf{I}-\mathbf{m} \otimes \mathbf{m})+S_{L} \mathbf{m} \otimes \mathbf{m}
$$

where $S_{T}$ is the constant residual stress in the isotropic plane and $S_{L}$ is the constant residual uniaxial stress along $\mathbf{m}$.

Necessary and sufficient conditions for strong ellipticity in the absence of residual stress are (Merodio and Ogden, 2003; Steigmann and Ogden, 2007):

$$
\mu_{L}>0, \quad \varphi>0, \quad \mu_{T}>0, \quad \lambda+2 \mu_{T}>0
$$

and

$$
\left|\lambda+\alpha+\mu_{L}\right|<\mu_{L}+\sqrt{\varphi\left(\lambda+2 \mu_{T}\right)}
$$

where

$$
\varphi=\lambda+4 \mu_{L}-2 \mu_{T}+2 \alpha+\beta
$$

We first assume the axis of transverse isotropy to coincide with the unit normal $\mathbf{k}\left(=\mathbf{e}_{3}\right)$ to the film, and later consider the case when the axis lies in the interfacial plane; the film material exhibits reflection symmetry with respect to the interface in both cases.

(a) Fibre axis orthogonal to the interfacial plane

In this case the plane-stress condition $(76)_{1}$, with $\mathbf{m}=\mathbf{k}$, yields

$$
\mathbf{S}_{R}=S \mathbf{1}
$$


with $S$ constant. Using $(93)_{2}$, a straightforward but involved calculation (Steigmann and Ogden, 2007) leads to

$$
a=-(\bar{\lambda}+\bar{\alpha}) \operatorname{div} \mathbf{v}
$$

where $\bar{\lambda}=\lambda / \varphi$ and $\bar{\alpha}=\alpha / \varphi$. Further,

$$
\mathcal{D}[\nabla \mathbf{v}]=\varphi\left\{\left[\bar{\lambda}-(\bar{\lambda}+\bar{\alpha})^{2}\right](\operatorname{div} \mathbf{v}) \mathbf{1}+\bar{\mu}_{T}\left[\nabla \mathbf{v}+(\nabla \mathbf{v})^{t}\right]\right\}
$$

where $\bar{\mu}_{T}=\mu_{T} / \varphi$, yielding

$$
\begin{aligned}
\mathcal{A} \boldsymbol{\delta} & =(\mathcal{D}[\boldsymbol{\delta} \otimes \mathbf{n}]) \mathbf{n} \\
& =\varphi\left\{\left[\bar{\lambda}-(\bar{\lambda}+\bar{\alpha})^{2}\right](\boldsymbol{\delta} \cdot \mathbf{n}) \mathbf{n}+\bar{\mu}_{T}[\boldsymbol{\delta}+(\boldsymbol{\delta} \cdot \mathbf{n}) \mathbf{n}]\right\}
\end{aligned}
$$

and hence

$$
\mathcal{A} \boldsymbol{\delta}=\mu_{T} \boldsymbol{\delta}
$$

in the case of Love waves. Substituting into (121), we conclude that the polarization $\boldsymbol{\delta}$ is an arbitrary vector in the interfacial plane and from (120) that

where

$$
\sqrt{1-s^{2}}=\epsilon\left(r s^{2}-\frac{S+\mu_{T}}{\mu_{s}}\right)
$$

$$
r=\rho_{\kappa} / \rho_{s}
$$

is the ratio of film density to substrate density. This is the relevant dispersion relation. To solve it we assume that $1-s^{2}=O\left(\epsilon^{2}\right)$ and derive the consistent estimate (Steigmann and Ogden, 2007)

$$
s \sim 1-\frac{1}{2} \epsilon^{2}\left(r-\frac{S+\mu_{T}}{\mu_{s}}\right)^{2}+o\left(\epsilon^{2}\right) .
$$

(b) Fibres lying in the interfacial plane

In this case $(76)_{1}$ yields

$$
\mathbf{S}_{R}=S \mathbf{m} \otimes \mathbf{m}
$$

a uniform uniaxial stress along the fibres, while $(93)_{2}$ gives

$$
a=-\frac{1}{\lambda+2 \mu_{T}}[\lambda \operatorname{div} \mathbf{v}+\alpha \mathbf{m} \cdot(\nabla \mathbf{v}) \mathbf{m}] .
$$

With some effort (95) and (96) may be reduced to (Steigmann, 2009a)

$$
\begin{aligned}
\mathcal{D}[\nabla \mathbf{v}]= & {[\lambda(\theta+a)+\alpha \mathbf{m} \cdot(\nabla \mathbf{v}) \mathbf{m}] \mathbf{I}+[\alpha(\theta+a)+\beta \mathbf{m} \cdot(\nabla \mathbf{v}) \mathbf{m}] \mathbf{m} \otimes \mathbf{m} } \\
& +2 \mu_{T}(\operatorname{Sym} \nabla \mathbf{v}+a \mathbf{k} \otimes \mathbf{k}) \\
& +2\left(\mu_{L}-\mu_{T}\right)[(\operatorname{Sym} \nabla \mathbf{v}) \mathbf{m} \otimes \mathbf{m}+\mathbf{m} \otimes(\operatorname{Sym} \nabla \mathbf{v}) \mathbf{m}] .
\end{aligned}
$$

For Love waves the latter is used to derive

$$
\begin{aligned}
\mathcal{A} \boldsymbol{\delta}= & (\mathcal{D}[\boldsymbol{\delta} \otimes \mathbf{n}]) \mathbf{n} \\
= & \alpha\left(1-\frac{\lambda}{\lambda+2 \mu_{T}}\right)(\mathbf{m} \cdot \mathbf{n})(\mathbf{m} \cdot \boldsymbol{\delta}) \mathbf{n}+\mu_{T} \boldsymbol{\delta} \\
& +\left(\beta-\frac{\alpha^{2}}{\lambda+2 \mu_{T}}\right)(\mathbf{m} \cdot \mathbf{n})^{2}(\mathbf{m} \cdot \boldsymbol{\delta})[(\mathbf{m} \cdot \mathbf{n}) \mathbf{n}+(\mathbf{m} \cdot \boldsymbol{\delta}) \boldsymbol{\delta}] \\
& +\left(\mu_{L}-\mu_{T}\right)\left[(\mathbf{m} \cdot \mathbf{n})^{2} \boldsymbol{\delta}+2(\mathbf{m} \cdot \mathbf{n})(\mathbf{m} \cdot \boldsymbol{\delta}) \mathbf{n}+(\mathbf{m} \cdot \boldsymbol{\delta})^{2} \boldsymbol{\delta}\right],
\end{aligned}
$$


and the propagation condition (121), projected onto the directions of $\boldsymbol{\delta}$ and $\mathbf{n}$, furnishes

$$
\begin{aligned}
(\mathbf{m} \cdot \mathbf{n})(\mathbf{m} \cdot \boldsymbol{\delta})\left[\delta(\mathbf{m} \cdot \mathbf{n})^{2}+\alpha\left(1-\frac{\lambda}{\lambda+2 \mu_{T}}\right)+2\left(\mu_{L}-\mu_{T}\right)\right] & =0 \text { and } \\
\epsilon\left[\rho_{\kappa} c^{2}-S(\mathbf{m} \cdot \mathbf{n})^{2}-\mu_{L}+\delta(\mathbf{m} \cdot \mathbf{n})^{2}(\mathbf{m} \cdot \boldsymbol{\delta})^{2}\right] & =\mu_{s} \eta,
\end{aligned}
$$

where

$$
\delta=\beta-\frac{\alpha^{2}}{\lambda+2 \mu_{T}} .
$$

Typical data on carbon fibre/epoxy composites furnish a non-zero value of the bracketed expression in the first relation, implying that

$$
(\mathbf{m} \cdot \mathbf{n})(\mathbf{m} \cdot \boldsymbol{\delta})=0
$$

and hence that either the direction of propagation or the polarization is parallel to the fibres.

In the first case we have $\mathbf{m} \cdot \mathbf{n}= \pm 1$ and $(139)_{2}$ delivers the associated dispersion relation

$$
\mu_{s} \eta=\epsilon\left(\rho_{\kappa} c^{2}-S-\mu_{L}\right)
$$

which is solved as before to obtain the estimate

$$
s \sim 1-\frac{1}{2} \epsilon^{2}\left(r-\frac{S+\mu_{L}}{\mu_{s}}\right)^{2}+o\left(\epsilon^{2}\right)
$$

In the second case $\mathbf{m}= \pm \boldsymbol{\delta}$ and the dispersion relation is

$$
\mu_{s} \eta=\epsilon\left(\rho_{\kappa} c^{2}-\mu_{L}\right)
$$

yielding

$$
s \sim 1-\frac{1}{2} \epsilon^{2}\left(r-\frac{\mu_{L}}{\mu_{s}}\right)^{2}+o\left(\epsilon^{2}\right) .
$$

We observe that in both cases the deformation is a shear, not in the isotropic plane, but rather in the plane containing the fibres. The operative material property is therefore $\mu_{L}$, the longitudinal shear modulus (Steigmann, 2010).

\subsection{Cubic symmetry}

In the case of cubic crystal symmetry we assume the cubic axes to be aligned with $\left\{\mathbf{e}_{i}\right\}=\left\{\mathbf{e}_{\alpha}, \mathbf{k}\right\}$. The residual stress is necessarily a pure pressure which vanishes by virtue of the plane-stress condition $(76)_{1}$. Accordingly, the strain energy $\bar{W}(\boldsymbol{\epsilon})$ is a homogeneous quadratic function which depends on the strain via the combinations (Smith et al, 1963)

$$
\left(\epsilon_{11}+\epsilon_{22}+\epsilon_{33}\right)^{2}, \quad \epsilon_{11} \epsilon_{22}+\epsilon_{11} \epsilon_{33}+\epsilon_{22} \epsilon_{33} \quad \text { and } \quad \epsilon_{12}^{2}+\epsilon_{13}^{2}+\epsilon_{23}^{2}
$$

These are common to all five subclasses of cubic symmetry.

It proves convenient to express the strain-energy function in terms of the spherical and deviatoric part of the strain, the latter being defined by

$$
\overline{\boldsymbol{\epsilon}}=\boldsymbol{\epsilon}-\frac{1}{3}(\operatorname{tr} \boldsymbol{\epsilon}) \mathbf{I}
$$


yielding

$$
\epsilon_{11} \epsilon_{22}+\epsilon_{11} \epsilon_{33}+\epsilon_{22} \epsilon_{33}=\frac{1}{3}(\operatorname{tr} \boldsymbol{\epsilon})^{2}-\frac{1}{2}\left(\bar{\epsilon}_{11}^{2}+\bar{\epsilon}_{22}^{2}+\bar{\epsilon}_{33}^{2}\right)
$$

and hence

$$
\bar{W}(\boldsymbol{\epsilon})=\frac{1}{2}\left[C_{1}\left(\epsilon_{11}+\epsilon_{22}+\epsilon_{33}\right)^{2}+C_{2}\left(\bar{\epsilon}_{11}^{2}+\bar{\epsilon}_{22}^{2}+\bar{\epsilon}_{33}^{2}\right)\right]+C_{3}\left(\epsilon_{12}^{2}+\epsilon_{13}^{2}+\epsilon_{23}^{2}\right),
$$

where $C_{1,2,3}$ are material constants. To ensure strong ellipticity in accordance with our assumptions thus far it is enough to require that $\bar{W}(\boldsymbol{\epsilon})$ be positive definite. Because (148) is the sum of independent quadratic forms this in turn is equivalent to the restrictions

$$
C_{1}>0, \quad C_{2}>0 \text { and } \quad C_{3}>0 .
$$

According to (73) the stress in the film is then given by

$$
\begin{aligned}
\mathbf{P}= & \mathcal{C}[\boldsymbol{\epsilon}] \\
= & C_{1}(\operatorname{tr} \boldsymbol{\epsilon}) \mathbf{I}+C_{2}\left(\bar{\epsilon}_{11} \mathbf{e}_{1} \otimes \mathbf{e}_{1}+\bar{\epsilon}_{22} \mathbf{e}_{2} \otimes \mathbf{e}_{2}+\bar{\epsilon}_{33} \mathbf{e}_{3} \otimes \mathbf{e}_{3}\right) \\
& +C_{3}\left[\epsilon_{12}\left(\mathbf{e}_{1} \otimes \mathbf{e}_{2}+\mathbf{e}_{2} \otimes \mathbf{e}_{1}\right)+\epsilon_{13}\left(\mathbf{e}_{1} \otimes \mathbf{e}_{3}+\mathbf{e}_{3} \otimes \mathbf{e}_{1}\right)+\epsilon_{23}\left(\mathbf{e}_{2} \otimes \mathbf{e}_{3}+\mathbf{e}_{3} \otimes \mathbf{e}_{2}\right)\right],
\end{aligned}
$$

and the plane-stress condition $(76)_{2}$ yields the restrictions

$$
C_{1}(t r \epsilon)+C_{2} \bar{\epsilon}_{33}=0 \quad \text { and } \quad \epsilon_{\alpha 3}=0
$$

on the interfacial values of the strain in the film.

Using (84) and (85) and the fact that $\boldsymbol{\epsilon}$ is the symmetric part of the displacement gradient, we have

$$
\boldsymbol{\epsilon}=\operatorname{Sym}(\nabla \mathbf{v})+a \mathbf{k} \otimes \mathbf{k}+\frac{1}{2}[\mathbf{k} \otimes(\nabla w+\boldsymbol{\alpha})+(\nabla w+\boldsymbol{\alpha}) \otimes \mathbf{k}],
$$

and thus conclude that $(151)_{2}$ is equivalent to (93) . Further, $a=\epsilon_{33}, \operatorname{tr} \boldsymbol{\epsilon}=\theta+a$, where $\theta=\operatorname{div} \mathbf{v}$, $\bar{\epsilon}_{33}=\frac{2}{3} a-\frac{1}{2} \theta$ and $(93)_{2}$ delivers

$$
a=-\left(C_{1}+\frac{2}{3} C_{2}\right)^{-1}\left(C_{1}-\frac{1}{3} C_{2}\right) \theta
$$

The plane-stress moduli are defined by (95) and (96) and hence given by

$$
\mathcal{D}[\nabla \mathbf{v}]=C_{1}(\theta+a) \mathbf{1}+C_{2}\left(\bar{\epsilon}_{11} \mathbf{e}_{1} \otimes \mathbf{e}_{1}+\bar{\epsilon}_{22} \mathbf{e}_{2} \otimes \mathbf{e}_{2}\right)+C_{3} \epsilon_{12}\left(\mathbf{e}_{1} \otimes \mathbf{e}_{2}+\mathbf{e}_{2} \otimes \mathbf{e}_{1}\right),
$$

where

$$
\bar{\epsilon}_{11}=\frac{1}{3 C_{1}+2 C_{2}}\left[\left(3 C_{1}+2 C_{2}\right) v_{1,1}+C_{2} v_{2,2}\right], \quad \bar{\epsilon}_{22}=\frac{1}{3 C_{1}+2 C_{2}}\left[\left(3 C_{1}+2 C_{2}\right) v_{2,2}+C_{2} v_{1,1}\right]
$$

and

$$
\theta+a=\frac{3 C_{2}}{3 C_{1}+2 C_{2}}\left(v_{1,1}+v_{2,2}\right) .
$$

To obtain $\mathcal{D}[\boldsymbol{\delta} \otimes \mathbf{n}]$ for use in (121) we simply replace $\nabla \mathbf{v}$ by $\boldsymbol{\delta} \otimes \mathbf{n}$, arriving at

$$
\begin{aligned}
\mathcal{A} \boldsymbol{\delta}= & (\mathcal{D}[\boldsymbol{\delta} \otimes \mathbf{n}]) \mathbf{n} \\
= & \frac{3 C_{1} C_{2}}{3 C_{1}+2 C_{2}}(\boldsymbol{\delta} \cdot \mathbf{n}) \mathbf{n}+\frac{C_{2}}{3 C_{1}+2 C_{2}}\left[\left(3 C_{1}+C_{2}\right) \delta_{1} n_{1}^{2}+C_{2} \delta_{2} n_{1} n_{2}\right] \mathbf{e}_{1} \\
& +\frac{C_{2}}{3 C_{1}+2 C_{2}}\left[\left(3 C_{1}+C_{2}\right) \delta_{2} n_{2}^{2}+C_{2} \delta_{1} n_{1} n_{2}\right] \mathbf{e}_{2} \\
& +\frac{1}{2} C_{3}\left(\delta_{1} n_{2}^{2}+\delta_{2} n_{1} n_{2}\right) \mathbf{e}_{1}+\frac{1}{2} C_{3}\left(\delta_{1} n_{1} n_{2}+\delta_{2} n_{1}^{2}\right) \mathbf{e}_{2} .
\end{aligned}
$$


An explicit expression for $\mathcal{A}$ follows by using $\delta_{\alpha}=\mathbf{e}_{\alpha} \cdot \boldsymbol{\delta}$ with $\delta_{1} \mathbf{e}_{1}=\left(\mathbf{e}_{1} \otimes \mathbf{e}_{1}\right) \boldsymbol{\delta}, \delta_{1} \mathbf{e}_{2}=\left(\mathbf{e}_{2} \otimes \mathbf{e}_{1}\right) \boldsymbol{\delta}$, etc.; thus, in the case of Love waves $(\boldsymbol{\delta} \cdot \mathbf{n}=0)$,

$$
\begin{aligned}
\mathcal{A}= & \left(C_{2} \frac{3 C_{1}+C_{2}}{3 C_{1}+2 C_{2}} n_{1}^{2}+\frac{1}{2} C_{3} n_{2}^{2}\right) \mathbf{e}_{1} \otimes \mathbf{e}_{1}+\left(C_{2} \frac{3 C_{1}+C_{2}}{3 C_{1}+2 C_{2}} n_{2}^{2}+\frac{1}{2} C_{3} n_{1}^{2}\right) \mathbf{e}_{2} \otimes \mathbf{e}_{2} \\
& +n_{1} n_{2}\left(\frac{C_{2}^{2}}{3 C_{1}+2 C_{2}}+\frac{1}{2} C_{3}\right)\left(\mathbf{e}_{1} \otimes \mathbf{e}_{2}+\mathbf{e}_{2} \otimes \mathbf{e}_{1}\right) .
\end{aligned}
$$

Recalling that the propagation condition (121) implies that $\mathbf{n}$ is an eigenvector, we have $\mathcal{A} \mathbf{n}=\xi \mathbf{n}$ for some $\xi \in \mathbb{R}$, where

$$
\begin{aligned}
\mathcal{A} \mathbf{n}= & \left(C_{2} \frac{3 C_{1}+C_{2}}{3 C_{1}+2 C_{2}} n_{1}^{2}+\frac{1}{2} C_{3} n_{2}^{2}\right) n_{1} \mathbf{e}_{1}+\left(C_{2} \frac{3 C_{1}+C_{2}}{3 C_{1}+2 C_{2}} n_{2}^{2}+\frac{1}{2} C_{3} n_{1}^{2}\right) n_{2} \mathbf{e}_{2} \\
& +n_{1} n_{2}\left(\frac{C_{2}^{2}}{3 C_{1}+2 C_{2}}+\frac{1}{2} C_{3}\right)\left(n_{2} \mathbf{e}_{1}+n_{1} \mathbf{e}_{2}\right) .
\end{aligned}
$$

Taking the scalar product of the equation $\mathcal{A} \mathbf{n}=\xi \mathbf{n}$ with $n_{2} \mathbf{e}_{1}$ and $n_{1} \mathbf{e}_{2}$ yields two equations for $\xi n_{1} n_{2}$, which we subtract to derive

$$
n_{1} n_{2}\left(n_{1}^{2}-n_{2}^{2}\right) \frac{3 C_{1}+C_{2}}{3 C_{1}+2 C_{2}}=0,
$$

and with (149) this yields the possibilities

$$
n_{1} n_{2}=0 \quad \text { or } \quad n_{1}^{2}=n_{2}^{2}
$$

The first alternative implies that $\mathbf{n} \in\left\{\mathbf{e}_{\alpha}\right\}$ and hence that $\boldsymbol{\delta} \in\left\{\mathbf{e}_{\alpha}\right\}$; the propagation and polarization directions are aligned with the crystallographic axes. Eq. (157) then provides

$$
\mathcal{A} \boldsymbol{\delta}=\frac{1}{2} C_{3} \boldsymbol{\delta}
$$

and (121) yields the dispersion relation

$$
\mu_{s} \eta=\epsilon\left(\rho_{\kappa} c^{2}-\frac{1}{2} C_{3}\right)
$$

which is solved as before to obtain

$$
s \sim 1-\frac{1}{2} \epsilon^{2}\left(r-\frac{C_{3}}{2 \mu_{s}}\right)^{2}+o\left(\epsilon^{2}\right) .
$$

The second alternative implies that $n_{1}^{2}=n_{2}^{2}=n^{2}$, with $n= \pm 1 / \sqrt{2}$. These yield the two families

$$
\begin{aligned}
& \mathbf{n}_{(1)}=\frac{1}{\sqrt{2}}\left(\mathbf{e}_{1}+\mathbf{e}_{2}\right), \quad \text { with } \quad \boldsymbol{\delta}_{(1)}=\frac{1}{\sqrt{2}}\left(\mathbf{e}_{1}-\mathbf{e}_{2}\right), \quad \text { and } \\
& \mathbf{n}_{(2)}=\frac{1}{\sqrt{2}}\left(\mathbf{e}_{1}-\mathbf{e}_{2}\right), \quad \text { with } \quad \boldsymbol{\delta}_{(2)}=\frac{1}{\sqrt{2}}\left(\mathbf{e}_{1}+\mathbf{e}_{2}\right),
\end{aligned}
$$

corresponding to propagation and polarization at 45 degrees to the crystallographic axes. In either case we have

$$
\mathcal{A}=\frac{1}{2}\left(C_{2} \frac{3 C_{1}+C_{2}}{3 C_{1}+2 C_{2}}+\frac{1}{2} C_{3}\right) \mathbf{1}+n_{1} n_{2}\left(\frac{C_{2}^{2}}{3 C_{1}+2 C_{2}}+\frac{1}{2} C_{3}\right)\left(\mathbf{e}_{1} \otimes \mathbf{e}_{2}+\mathbf{e}_{2} \otimes \mathbf{e}_{1}\right)
$$

implying that

$$
\mathcal{A} \boldsymbol{\delta}=\frac{3}{2} \frac{C_{1} C_{2}}{3 C_{1}+2 C_{2}} \boldsymbol{\delta} .
$$

Finally, substitution into (121) furnishes the dispersion relation

$$
\mu_{s} \eta=\epsilon\left(\rho_{\kappa} c^{2}-\frac{3}{2} \frac{C_{1} C_{2}}{3 C_{1}+2 C_{2}}\right)
$$


and thus

$$
s \sim 1-\frac{1}{2} \epsilon^{2}\left[r-\frac{3}{2 \mu_{s}} \frac{C_{1} C_{2}}{\left(3 C_{1}+2 C_{2}\right)}\right]^{2}+o\left(\epsilon^{2}\right) .
$$

\section{Survey of nonlinear and linearized electroelasticity}

Here we review the basic theory of nonlinear electroelasticity as a prelude to the development of a model for thin electro-elastic films. For further background reference may be made to (Eringen and Maugin, 1990; Kovetz, 2000; Steigmann, 2009b). In nonlinear electroelasticity we assume the existence of a free energy per unit mass, $\varphi$ say, that depends on the deformation gradient $\mathbf{F}$ and electric field $\mathbf{e}$. Here we restrict attention to the purely electromechanical theory and suppress thermal and electrical conduction. We also assume the material to be non-magnetizable.

The Cauchy stress is (Kovetz, 2000)

$$
\mathbf{T}=\rho \varphi_{\mathbf{F}} \mathbf{F}^{t}+\mathbf{T}_{M}
$$

where

$$
\mathbf{T}_{M}=\varepsilon_{0}\left(\mathbf{e} \otimes \mathbf{e}-\frac{1}{2} e^{2} \mathbf{I}\right), \quad \text { with } \quad e=|\mathbf{e}|,
$$

is the Maxwell stress in which $\varepsilon_{0}$ is the free-space permittivity. The material polarization is

$$
\mathbf{p}=-\rho \varphi_{\mathbf{e}}
$$

By an argument similar to that leading from (3) to (23) (Kovetz, 2000), we find that the free energy depends on the deformation and electric field via the Cauchy-Green deformation tensor $\mathbf{C}=\mathbf{F}^{t} \mathbf{F}$ and the pullback $\mathbf{E}=\mathbf{F}^{t} \mathbf{e}$; thus,

$$
\varphi(\mathbf{F}, \mathbf{e})=\Phi(\mathbf{C}, \mathbf{E}) .
$$

Straightforward application of the chain rule yields

$$
\varphi_{\mathbf{e}}=\mathbf{F} \Phi_{\mathbf{E}} \quad \text { and } \quad \varphi_{\mathbf{F}}=2 \mathbf{F} \Phi_{\mathbf{C}}+\mathbf{e} \otimes \Phi_{\mathbf{E}}
$$

and hence

$$
\mathbf{T}=\boldsymbol{\sigma}+\mathbf{T}_{M},
$$

where

$$
\boldsymbol{\sigma}=2 \rho \mathbf{F} \Phi_{\mathbf{C}} \mathbf{F}^{t}
$$

together with

$$
\mathbf{p}=-\rho \mathbf{F} \Phi_{\mathbf{E}} .
$$

In the absence of a magnetic induction field or volumetric distributions of charge, the equations to be solved are

$$
\operatorname{div} \mathbf{T}=\rho \boldsymbol{\chi}_{t t}, \quad \operatorname{curl} \mathbf{e}=\mathbf{0} \quad \text { and } \quad \operatorname{div} \mathbf{d}=0
$$

where

$$
\mathbf{d}=\varepsilon_{0} \mathbf{e}+\mathbf{p}
$$


is the electric displacement and curl is the spatial curl operator, together with $\operatorname{curl} \mathbf{h}=\mathbf{0}$, where $\mathbf{h}$ is the magnetic field. The latter is valid in the absence of currents under the so-called quasi-electrostatic approximation (Eringen and Maugin, 1990), according to which time derivatives appearing in Maxwell's equations are negligible compared to time derivatives occurring in the equation of motion. Further, for non-magnetizable bodies it is possible to show that $\mathbf{h}=\boldsymbol{\chi}_{t} \times \mathbf{p}$. This is a nonlinear term and hence negligible in the linear theory to be discussed; the restriction $\operatorname{curl} \mathbf{h}=\mathbf{0}$ is then effectively reduced to an identity and plays no role in the linear theory.

Appended to this system are the boundary conditions

$$
\mathbf{t}_{a}+\mathbf{T}_{M}^{+} \mathbf{n}=\mathbf{T n}, \quad \mathbf{n} \cdot[\mathbf{d}]=\sigma \quad \text { and } \quad \mathbf{n} \times[\mathbf{e}]=\mathbf{0},
$$

the first applying on a part of the boundary where the applied traction $\mathbf{t}_{a}$ is specified, where $\mathbf{n}$ is the exterior unit normal to the boundary, $\sigma$ is the areal density of surface charge on the boundary, and where $[\cdot]=(\cdot)^{+}-(\cdot)^{-}$, with the superscripts \pm referring respectively to limits as the boundary is approached from the exterior and interior of the body.

We require the referential forms of the equations, expressed in terms of differential operators with respect to $\mathbf{x}$. To derive the relevant version of $(178)_{2}$, we use Stokes' theorem

$$
\int_{s} \mathbf{n} \cdot \operatorname{curl} \mathbf{e} d a=\int_{\partial s} \mathbf{e} \cdot d \mathbf{y}=\int_{\partial S} \mathbf{e} \cdot \mathbf{F} d \mathbf{x}=\int_{\partial S} \mathbf{E} \cdot d \mathbf{x}=\int_{S} \mathbf{N} \cdot C u r l \mathbf{E} d A,
$$

where $S$ is an arbitrary material surface with $s=\chi(S, t)$ its image in the current configuration, and Curl is the referential curl operator, to conclude that $(178)_{2}$ is equivalent to

$$
\operatorname{Curl} \mathbf{E}=\mathbf{0}
$$

which implies that

$$
\mathbf{E}=-G r a d V
$$

for some scalar potential $V$. A slight generalization of the argument delivers the referential version of the jump condition $(180)_{3}$ :

$$
\mathbf{N} \times[\mathbf{E}]=\mathbf{0} .
$$

In the same way we use the divergence theorem to deduce that, for an arbitrary material subvolume $\pi$ with $P=\chi(\pi, t)$,

$$
\int_{P} \operatorname{div} \mathbf{d} d v=\int_{\partial P} \mathbf{d} \cdot \mathbf{n} d a=\int_{\partial \pi} \mathbf{d} \cdot \mathbf{F}^{*} \mathbf{N} d A=\int_{\partial \pi} J \mathbf{F}^{-1} \mathbf{d} \cdot \mathbf{N} d A=\int_{\pi} \operatorname{Div}\left(J \mathbf{F}^{-1} \mathbf{d}\right) d V,
$$

and thus that $(178)_{3}$ is equivalent to

$$
\operatorname{Div} \mathbf{D}=0, \quad \text { where } \quad \mathbf{D}=J \mathbf{F}^{-1} \mathbf{d} .
$$

Again the procedure may be generalized to derive the appropriate version of $(180)_{2}$ :

$$
\mathbf{N} \cdot[\mathbf{D}]=\Sigma,
$$

where $\Sigma=\sigma\left|\mathbf{F}^{*} \mathbf{N}\right|$ is the referential surface charge density. 
A convenient definition of the referential polarization is

$$
\Pi=J \mathbf{F}^{-1} \mathbf{p} .
$$

This yields (cf. (177))

$$
\Pi=-\rho_{\kappa} \Phi_{\mathbf{E}}
$$

and thus (cf. (179))

$$
\mathbf{D}=\boldsymbol{\Pi}+\varepsilon_{0} J \mathbf{C}^{-1} \mathbf{E}
$$

Lastly, the referential equation of motion is given precisely by (2) in which $\mathbf{T}$ is now given by (170) or (175). Then,

$$
\mathbf{P}=\left(\boldsymbol{\sigma}+\mathbf{T}_{\mathbf{M}}\right) \mathbf{F}^{*}=2 \mathbf{F} W_{\mathbf{C}}+\mathbf{T}_{M} \mathbf{F}^{*}
$$

with

$$
\mathbf{T}_{M} \mathbf{F}^{*}=\varepsilon_{0} \mathbf{F}^{*}\left[\mathbf{E} \otimes\left(\mathbf{C}^{-1} \mathbf{E}\right)-\frac{1}{2} e^{2} \mathbf{I}\right],
$$

and where $W=\rho_{\kappa} \Phi$ is the strain-energy function.

To linearize the theory we suppose the norms of the electric field and the displacement gradient to be small and use the strain measure defined by (25) to obtain

$$
\begin{aligned}
\boldsymbol{\sigma} \mathbf{F}^{*} & =\mathbf{F} \bar{W}_{\boldsymbol{\epsilon}} \\
& =(\mathbf{I}+\mathbf{H})\{\mathcal{C}[\boldsymbol{\epsilon}]+\mathcal{S} \mathbf{E}+\ldots\} \\
& \simeq \mathcal{C}[\mathbf{H}]+\mathbf{S E}
\end{aligned}
$$

where $\bar{W}$ is the strain energy expressed as a function of $\boldsymbol{\epsilon}$ and $\mathbf{E}$, and $\mathcal{C}$ and $\mathcal{S}$ respectively are the values of $\bar{W}_{\boldsymbol{\epsilon} \epsilon}$ and $\bar{W}_{\mathbf{E} \epsilon}$ when the strain and electric field vanish. Here and henceforth, for convenience, we assume that the associated values of stress and polarization vanish. Thus we assume the absence of residual stress and residual polarization. To linear order we also have

$$
\mathbf{P} \simeq \sigma \mathbf{F}^{*}
$$

since the Maxwell stress is quadratic in the electric field.

In the same way we have

$$
-\mathbf{\Pi}=\bar{W}_{\mathbf{E}} \simeq \mathcal{Q} \mathbf{E}+\mathcal{R} \boldsymbol{\epsilon},
$$

where $\mathcal{Q}$ and $\mathcal{R}$ respectively are the values of $\bar{W}_{\mathbf{E E}}$ and $\bar{W}_{\boldsymbol{\epsilon} \mathbf{E}}$ at zero strain and electric field. Combining this with (190) and $J \mathbf{C}^{-1} \mathbf{E} \simeq \mathbf{E}$ we obtain

$$
\mathbf{D} \simeq \varepsilon_{0} \mathbf{E}-\bar{W}_{\mathbf{E}}
$$

In component form, eqs. (5) and (186) are given, to linear order, by

$$
\rho_{\kappa} u_{i t t}=\mathcal{C}_{i j k l} u_{k, j l}+\mathcal{S}_{i j k} E_{k, j}
$$

and

$$
\left[\left(\varepsilon_{0} \delta_{i j}-Q_{i j}\right) E_{j}\right]_{, i}=\mathcal{S}_{i j k} u_{j, k i},
$$


where

$$
\mathcal{S}_{i j k}=\partial^{2} \bar{W} / \partial E_{k} \partial \epsilon_{i j},
$$

$u_{i}$ are the displacement components, $E_{i}=-V_{, i}$ and commas followed by subscripts are used to denote partial derivatives with respect to the initial Cartesian coordinates $x_{i}$.

Relevant to our analysis of thin-film substrate problems are restrictions on the various moduli ensuring the existence of propagating plane harmonic waves. To explore this we consider plane harmonic bulk waves of the form

$$
u_{i}=a_{i} \exp [i(\mathbf{k} \cdot \mathbf{x}-\omega t)], \quad V=v \exp [i(\mathbf{k} \cdot \mathbf{x}-\omega t)]
$$

where $a_{i}$ are the (constant) components of the displacement polarization, $v$ is a constant, $\omega$ is the frequency and $\mathbf{k}$ is the wave vector. The direction of propagation is $\mathbf{n}$ and $\mathbf{k}=k \mathbf{n}$, where $k$ is the wave number; the wavespeed $c$ is then given by $\omega=k c$. Substitution into (197) and (198) furnishes the algebraic system

$$
-\rho_{\kappa} \omega^{2} u_{i}=-\mathcal{C}_{i j k l} k_{j} k_{l} u_{k}+\mathcal{S}_{i j k} k_{j} k_{k} V \quad \text { and } \quad \mathcal{S}_{i j k} k_{j} k_{k} u_{j}+\eta_{i j} k_{i} k_{j} V=0
$$

where

$$
\eta_{i j}=\varepsilon_{0} \delta_{i j}-Q_{i j}
$$

We assume that $\boldsymbol{\eta}$ is non-singular so that $\mathbf{k} \cdot \boldsymbol{\eta} \mathbf{k} \neq 0$ for any non-zero $\mathbf{k}$. Then,

$$
V=-(\mathbf{k} \cdot \boldsymbol{\eta} \mathbf{k})^{-1} \mathcal{S}_{i j k} k_{j} k_{k} u_{j}
$$

and

$$
\rho_{\kappa} c^{2} u_{i}=\left[A_{i j}+(\mathbf{k} \cdot \boldsymbol{\eta} \mathbf{k})^{-1} \Gamma_{i} \Gamma_{j}\right] u_{j}
$$

where

$$
A_{i j}=\mathcal{C}_{i j k l} n_{k} n_{l} \quad \text { and } \quad \Gamma_{i}=\mathcal{S}_{i j k} n_{j} n_{k} .
$$

Accordingly, sufficient conditions for propagation are the positivity of the tensors $\mathbf{A}$ and $\boldsymbol{\eta}$ (Baesu et al, 2003), the former generalizing the classical propagation condition of conventional elasticity theory.

\section{Thin-film model}

Suppose the film is coated with a very thin layer of perfectly conducting electrode material carrying a charge density $\Sigma$. We assume that the substrate to which the film is attached is also a perfect conductor. Then the electric and polarization fields exterior to the film vanish. The jump conditions $(180)_{2,3}$, applied at the interfaces between the film and the electrode and substrate, then yield

$$
\mathbf{n} \cdot \mathbf{d}^{(i)}=-\sigma \quad \text { and } \quad \mathbf{n} \times \mathbf{e}^{(i)}=\mathbf{0}
$$

where the superscript $(i)$ refers to the limit as the interface is approached from the interior of the film. The associated referential forms are

$$
\mathbf{N} \cdot \mathbf{D}^{(i)}=-\Sigma \quad \text { and } \quad \mathbf{N} \times \mathbf{E}^{(i)}=\mathbf{0} .
$$


To make the first of these explicit we write $\mathbf{N} \cdot \mathbf{D}^{+}=-\Sigma^{+}$and $\mathbf{N} \cdot \mathbf{D}_{0}=-\Sigma_{0}$, these being respectively equal to the surface charges at the electrode/film interface and the film/substrate interface where $\mathbf{N}= \pm \mathbf{k}$.

The weak form of the equation of motion in the film material is given by (38), but with the stress $\mathbf{P}$ now given by (194). The weak form of (186) is given by

$$
\int_{\pi} \mathbf{D} \cdot G r a d \dot{V} d V=\int_{\partial \pi} \mathbf{D} \cdot \mathbf{N} \dot{V} d A
$$

in which $\dot{V}$ is the variation of the electric potential and the integrand on the right-hand side is the limit from the interior of the film.

Proceeding as in (47) we decompose the electric displacement into a part in the interfacial plane and a part orthogonal to it, i.e.

$$
\mathbf{D}=\mathbf{1} \mathbf{D}+D \mathbf{k}, \quad \text { with } \quad D=\mathbf{k} \cdot \mathbf{D} .
$$

Thus,

$$
\int_{\partial \pi} \mathbf{D} \cdot \mathbf{N} \dot{V} d A=\int_{\partial \omega}\left(\int_{0}^{h} \mathbf{1} \mathbf{D} \cdot \boldsymbol{\nu} \dot{V} d \varsigma\right) d S+\int_{\omega^{+}} D^{+} \dot{V}^{+} d A-\int_{\omega} D_{0} \dot{V}_{0} d A
$$

where $\omega \subset \Omega$ is an arbitrary part of the film/substrate interface $\Omega$ and $\omega^{+}$is its projection onto the electrode/film interface. Using (207) ${ }_{1}$ and the estimate (44) we derive

$$
\int_{\partial \pi} \mathbf{D} \cdot \mathbf{N} \dot{V} d A=h \int_{\partial \omega} \mathbf{1 D}_{0} \cdot \boldsymbol{\nu} \dot{V}_{0} d S-\int_{\omega^{+}} \Sigma^{+} \dot{V}^{+} d A-\int_{\omega} \Sigma_{0} \dot{V}_{0} d A+o(h) .
$$

This is further reduced, using

$$
V^{+}=V_{0}+h V_{0}^{\prime}+o(h)
$$

to obtain

$$
\int_{\partial \pi} \mathbf{D} \cdot \mathbf{N} \dot{V} d A=-\int_{\omega}\left(\Sigma_{0}+\Sigma^{+}\right) \dot{V}_{0} d A+h \int_{\partial \omega} \mathbf{1 D}_{0} \cdot \boldsymbol{\nu} \dot{V}_{0} d S-h \int_{\omega} \Sigma^{+} \dot{V}_{0}^{\prime} d A+o(h) .
$$

In the same way,

$$
\int_{\pi} \mathbf{D} \cdot G r a d \dot{V} d V=h \int_{\omega}\left(\mathbf{1 D}_{0} \cdot \nabla \dot{V}_{0}+D_{0} \dot{V}_{0}^{\prime}\right) d A+o(h)
$$

having used the decomposition

$$
G r a d \dot{V}=\nabla \dot{V}+\dot{V}^{\prime} \mathbf{k}
$$

and the balance law (208) yields

$$
-\int_{\omega}\left(\Sigma_{0}+\Sigma^{+}\right) \dot{V}_{0} d A+h\left(\int_{\partial \omega} \mathbf{1 D}_{0} \cdot \boldsymbol{\nu} \dot{V}_{0} d S-\int_{\omega} \Sigma^{+} \dot{V}_{0}^{\prime} d A\right)=h \int_{\omega}\left(\mathbf{1 D}_{0} \cdot \nabla \dot{V}_{0}+D_{0} \dot{V}_{0}^{\prime}\right) d A+o(h) .
$$

We have implicitly imposed (182) and $(207)_{2}$ as constraints, the latter in the form $\mathbf{k} \times \mathbf{E}^{(i)}=\mathbf{0}$. Thus, $\operatorname{Curl} \dot{\mathbf{E}}=\mathbf{0}$ and $\mathbf{k} \times \dot{\mathbf{E}}^{(i)}=\mathbf{0}$, implying that $\dot{\mathbf{E}}=-G r a d \dot{V}$ with $\mathbf{k} \times G r a d \dot{V}^{(i)}=\mathbf{0}$ at the interfaces. From (215) we then have $\mathbf{k} \times \nabla \dot{V}_{0}=\mathbf{0}$ in particular, implying that $\dot{V}_{0}$ is uniform on $\Omega$. On $\Omega^{+}$we have

$$
\mathbf{0}=\mathbf{k} \times \nabla \dot{V}^{+}=\mathbf{k} \times \nabla \dot{V}_{0}+h \mathbf{k} \times \nabla \dot{V}_{0}^{\prime}+o(h)
$$

yielding

$$
\nabla \dot{V}_{0}^{\prime}=h^{-1} o(h)
$$


and hence

$$
-\dot{V}_{0} \int_{\omega}\left(\Sigma_{0}+\Sigma^{+}\right) d A+h \dot{V}_{0} \int_{\partial \omega} \mathbf{1 D}_{0} \cdot \boldsymbol{\nu} d S-h \dot{V}_{0}^{\prime} \int_{\omega}\left(D_{0}+\Sigma^{+}\right) d A+o(h)=0 .
$$

Because $\dot{V}_{0}$ and $\omega$ are arbitrary it follows that $\Sigma_{0}+\Sigma^{+}=O(h)$. We satisfy this by imposing

$$
\Sigma^{+}=-\Sigma_{0}
$$

leaving

$$
\dot{V}_{0} \int_{\partial \omega} \mathbf{1 D}_{0} \cdot \boldsymbol{\nu} d S-\dot{V}_{0}^{\prime} \int_{\omega}\left(D_{0}+\Sigma^{+}\right) d A+h^{-1} o(h)=0 .
$$

Passing to the limit and invoking the arbitrariness and independence of $\dot{V}_{0}$ and $\dot{V}_{0}^{\prime}$ results in

$$
\int_{\omega} \operatorname{div}\left(\mathbf{1 D}_{0}\right) d A=0 \quad \text { and } \quad \int_{\omega}\left(D_{0}-\Sigma_{0}\right) d A=0
$$

and the arbitrariness of $\omega$ finally yields

$$
\operatorname{div}\left(\mathbf{1 D}_{0}\right)=0 \text { and } D_{0}=\Sigma_{0}
$$

pointwise on $\Omega$.

Under the stated conditions the Maxwell stress exterior to the film vanishes. The traction boundary condition $(180)_{1}$ is then identical in form to $(4)$, after making the appropriate adjustment in the definition of the stress. The procedure used in Section 3 to derive the equation of motion for the film/substrate interface carries over unchanged and culminates in (55) in the case when the upper surface of the film is traction free.

\section{Linear theory for cubic symmetry: Love modes and extinguished waves}

In the linear approximation the constitutive equations are

$$
\mathbf{P}=\bar{W}_{\boldsymbol{\epsilon}} \quad \text { and } \quad \mathbf{D}=\varepsilon_{0} \mathbf{E}-\bar{W}_{\mathbf{E}}
$$

and to make these explicit we require an expression for $\bar{W}(\boldsymbol{\epsilon}, \mathbf{E})$ that is homogeneous of degree two.

Here we consider various subclasses of cubic symmetry. For example, the strain-energy function pertaining to the hextetrahedral subgroup is (Smith et al, 1963)

$$
\begin{aligned}
\bar{W}(\boldsymbol{\epsilon}, \mathbf{E})= & \frac{1}{2}\left[C_{1}\left(\epsilon_{11}+\epsilon_{22}+\epsilon_{33}\right)^{2}+C_{2}\left(\bar{\epsilon}_{11}^{2}+\bar{\epsilon}_{22}^{2}+\bar{\epsilon}_{33}^{2}\right)\right]+C_{3}\left(\epsilon_{12}^{2}+\epsilon_{13}^{2}+\epsilon_{23}^{2}\right) \\
& +\frac{1}{2} D_{1}\left(E_{1}^{2}+E_{2}^{2}+E_{3}^{2}\right)+2 D_{2}\left(E_{1} \epsilon_{23}+E_{2} \epsilon_{13}+E_{3} \epsilon_{12}\right), .
\end{aligned}
$$

where $C_{1,2,3}$ and $D_{1,2}$ are material parameters and we impose inequalities (149) to ensure that a sufficient condition for propagation, discussed in Section 6, is satisfied. The associated stress is

$$
\begin{aligned}
\mathbf{P}= & C_{1}(\operatorname{tr} \boldsymbol{\epsilon}) \mathbf{I}+C_{2}\left(\bar{\epsilon}_{11} \mathbf{e}_{1} \otimes \mathbf{e}_{1}+\bar{\epsilon}_{22} \mathbf{e}_{2} \otimes \mathbf{e}_{2}+\bar{\epsilon}_{33} \mathbf{e}_{3} \otimes \mathbf{e}_{3}\right) \\
& +\left(C_{3} \epsilon_{12}+D_{1} E_{3}\right)\left(\mathbf{e}_{1} \otimes \mathbf{e}_{2}+\mathbf{e}_{2} \otimes \mathbf{e}_{1}\right)+\left(C_{3} \epsilon_{13}+D_{1} E_{2}\right)\left(\mathbf{e}_{1} \otimes \mathbf{e}_{3}+\mathbf{e}_{3} \otimes \mathbf{e}_{1}\right) \\
& +\left(C_{3} \epsilon_{23}+D_{1} E_{1}\right)\left(\mathbf{e}_{2} \otimes \mathbf{e}_{3}+\mathbf{e}_{3} \otimes \mathbf{e}_{2}\right),
\end{aligned}
$$


and the electric displacement is

$$
\mathbf{D}=\left(\varepsilon_{0}-D_{1}\right) \mathbf{E}-2 D_{2}\left(\epsilon_{23} \mathbf{e}_{1}+\epsilon_{13} \mathbf{e}_{2}+\epsilon_{12} \mathbf{e}_{3}\right) .
$$

Equation $(223)_{2}$ then furnishes the restriction $\Sigma_{0}=\left(\varepsilon_{0}-D_{1}\right) E_{3}-2 D_{2} \epsilon_{12}$, implying that $E_{3}\left(=-V_{0}^{\prime}\right)$ satisfies

$$
\left(\varepsilon_{0}-D_{1}\right) E_{3}=\Sigma_{0}+2 D_{2} \epsilon_{12}
$$

whereas the restriction $(55)_{1}$ on the film stress at the film/substrate interface reduces to

$$
\mathbf{0}=\mathbf{P}_{0} \mathbf{k}=\left[C_{1}(\operatorname{tr} \boldsymbol{\epsilon})+C_{2} \bar{\epsilon}_{33}\right] \mathbf{k}+\left(C_{3} \epsilon_{13}+D_{1} E_{2}\right) \mathbf{e}_{1}+\left(C_{3} \epsilon_{23}+D_{1} E_{1}\right) \mathbf{e}_{2},
$$

yielding

$$
\epsilon_{13}=-\left(D_{1} / C_{3}\right) E_{2} \quad \text { and } \quad \epsilon_{23}=-\left(D_{1} / C_{3}\right) E_{1} .
$$

Recalling that $1 \mathrm{E}=-\nabla V_{0}$ at the interface, and hence that $E_{\alpha}=-V_{0, \alpha}=0$, we conclude that $\epsilon_{3 \alpha}=0$ in the film at the interface, as in the purely elastic theory. Further, $E_{3}$ is uniform at the interfacial plane because $\nabla V_{0}^{\prime}$ vanishes. Thus, if the assigned surface charge $\Sigma_{0}$ is uniform, and if $D_{2} \neq 0$, then (228) implies that $\epsilon_{12}$ is uniform on $\Omega$. This situation pertains not only to the hextetrahedral subgroup of the cubic symmetry group, but also to the tetardoidal and gyroidal subgroups (Smith et al, 1963). In contrast, $D_{2}=0$ in higher-symmetry materials characterized by the hexoctahedral and diploidal subgroups, and so for these there is no requirement that $\epsilon_{12}$ be uniform.

Proceeding, we have

$$
\mathbf{1} \mathbf{D}_{0}=\left(\varepsilon_{0}-D_{1}\right) \mathbf{1} \mathbf{E}-2 D_{2}\left(\epsilon_{23} \mathbf{e}_{1}+\epsilon_{13} \mathbf{e}_{2}\right),
$$

which vanishes identically, ensuring that $(223)_{1}$ is automatically satisfied. The equation of motion for the film/substrate interface is

$$
\boldsymbol{\sigma}_{0} \mathbf{k}=h\left[\operatorname{div}\left(\mathbf{P}_{0} \mathbf{1}\right)-\rho_{\kappa} \mathbf{u}_{0 t t}\right]
$$

in which $\boldsymbol{\sigma}$ is the stress in the substrate, assumed to be an isotropic, non-polarizable elastic solid, and

$$
\mathbf{P}_{0} \mathbf{1}=C_{1}(t r \boldsymbol{\epsilon}) \mathbf{1}+C_{2}\left(\bar{\epsilon}_{11} \mathbf{e}_{1} \otimes \mathbf{e}_{1}+\bar{\epsilon}_{22} \mathbf{e}_{2} \otimes \mathbf{e}_{2}\right)+\left(C_{3} \epsilon_{12}+D_{1} E_{3}\right)\left(\mathbf{e}_{1} \otimes \mathbf{e}_{2}+\mathbf{e}_{2} \otimes \mathbf{e}_{1}\right) .
$$

Our results yield

$$
\operatorname{div}\left\{E_{3}\left(\mathbf{e}_{1} \otimes \mathbf{e}_{2}+\mathbf{e}_{2} \otimes \mathbf{e}_{1}\right)\right\}=\mathbf{0},
$$

implying that (232) reduces to the purely elastic problem treated in Section 5. Accordingly a uniform surface charge on a polarizable cubic crystal film has no effect on propagating waves.

However, we have seen that $\nabla \epsilon_{12}=\mathbf{0}$ on $\Omega$ if the film properties are such that $D_{2} \neq 0$. For Love waves, the in-plane displacement gradient is (cf. (105) 1 ) $\nabla \mathbf{v}=i k G \boldsymbol{\delta} \otimes \mathbf{n}$, with $\boldsymbol{\delta} \cdot \mathbf{n}=0$. Writing $\mathbf{n}=\cos \theta \mathbf{e}_{1}+\sin \theta \mathbf{e}_{2}$ and $\boldsymbol{\delta}=-\sin \theta \mathbf{e}_{1}+\cos \theta \mathbf{e}_{2}$, we use this to derive

$$
2 \nabla \epsilon_{12}=-k^{2} G\left(\cos ^{2} \theta-\sin ^{2} \theta\right) \mathbf{n}
$$

and thus conclude that $\cos ^{2} \theta=\sin ^{2} \theta$, yielding $\theta= \pm 45^{\circ}$. This implies that waves propagating along the crystallographic axes are extinguished by the application of a uniform surface charge in polarizable cubic 
films belonging to the hextetrahedral, tetardoidal or gyroidal subclasses. We know of no experimental corroboration of this remarkable and potentially useful prediction.

\section{References}

S.S. Antman, Nonlinear Problems of Elasticity. Springer, Berlin, 2005.

E. Baesu, D. Fortune and E. Soós, Incremental behaviour of hyperelastic dielectrics and piezoelectric crystals. ZAMP 54:160-178, 2003.

P.G. Ciarlet, Mathematical Elasticity, Vol. 1: Three-Dimensional Elasticity. North-Holland, Amsterdam, 1988.

A.C. Eringen and G.A. Maugin, Electrodynamics of Continua, Vol. 1. Springer, N.Y., 1990.

A.E. Green and W. Zerna, Theoretical Elasticity, 2nd edn. Oxford University Press, 1968.

Y.B Fu, Linear and nonlinear wave propagation in coated or uncoated elastic half spaces. Waves in Nonlinear Pre-Stressed Materials (M. Destrade \& G. Saccomandi, Eds). CISM Courses and Lecture Notes. Springer, Wien, 2007.

A. Kovetz, Electromagnetic Theory. Oxford University Press, 2000.

J. Merodio and R.W. Ogden, A note on strong ellipticity for transversely isotropic linearly elastic solids. Q.J. Mech. Appl. Math. 56:589-591, 2003.

R.W. Ogden, Non-linear Elastic Deformations. Dover, N.Y., 1997.

G.F. Smith, M.M. Smith and R.S. Rivlin, Integrity bases for a symmetric tensor and a vector: The crystal classes. Arch. Ration. Mech. Anal. 12:93-133, 1963.

A.J.M. Spencer, Constitutive theory for strongly anisotropic solids, in: Continuum Theory of the Mechanics of Fibre-Reinforced Composites (A.J.M. Spencer, Ed.). CISM Courses and Lectures No. 282, pp. 1-32. Springer, Wien, 1984.

D.J. Steigmann and R.W. Ogden, Surface waves supported by thin-film/substrate interactions. IMA J. Appl. Math. 72:730-47, 2007.

D.J. Steigmann, Linear theory for the bending and extension of a thin, residually stressed, fiberreinforced lamina. Int. J. Engng. Sci. 47:1367-78, 2009a.

D.J. Steigmann, On the formulation of balance laws for electromagnetic continua. Math. Mech. Solids 14:390-402, 2009b.

D.J. Steigmann, Elastic waves interacting with a thin, pre-stressed, fiber-reinforced surface film. Int. J. Engng. Sci. 48:1604-09, 2010. 